\title{
VALORI MEDIANI MENSILI DELLE CARATTERISTICHE IONOSFERICHE A ROMA NEL PERIODO MARZO 1949 - APRILE 1953
}

\author{
Piero Dominici
}

Nelle tabelle che seguono sono riportati i valori mediani mensili delle frequenze critiche e delle altezze virtuali di riflessione delle regioni $F 2, F 1, E$ e le percentuali di apparizione dello strato $E$ sporadico ( $E s)$, calcolati sui valori orari forniti dai sondaggi ionosferici eflettuati dalla stazione ionosferica di Roma dell'Istituto Nazionale di Geofisica.

Sino al mese di marzo 1952 fu impiegato un apparato automatico di rẹi-lrazione che aveva lo sequenti caratterietiche $1 *$ :

Gamma di frequenza: 1 a 14 Mhz, continua;

Durata degli impulsi: 100 microsecondi;

Regristrazione: oscillografo a raggi catodici, modulazione $Z$. pellicola cinematografica $35 \mathrm{~mm}$;

Potenza media nella gamma di frequenza indicata: $10 \mathrm{KW}$ di cresta;

Antenne: rombica verticale in trasmissione;

dipolo orizzontale in ricezione;

A partire dal mese di novembre $1952 \mathrm{fu}$ sostituito a questo un altro apparato di migliorate caratteristiche costruttive e funzionali, con una gamma di frequenza più ampia ( 1 a 13 Mhz).

La durata delle registrazioni orarie è stata sempre di -10 secondi. Dal gennaio 1951 si è effettuata una registrazione nel primo minuto esatto di ogni ora: precedentemente le registrazioni erano 16 al giorno (12 per i mesi di marzo e aprile 1949) anziché 24, distribuite in modo da addensarsi intorno all'ora dell'alla e del tramonto.

Tempo medio dell'Europa centrale ( $\left.15^{\circ} \mathrm{E}\right)$.

Convenzioni adottate per il calcolo dei valori mediani mensili e simbologia usata. - I simboli che compaiono nelle tabelle hanno il seguente significato:

foF2: frequenza critica per il raggio ordinario, per la regione $F 2$.

(*) A. Bolle. "Annali di Geofision ", vol. 1. pạ. 16+-17t (1948). 
$h^{\prime} F 2$ : altezza virtuale minima di riflessione della regrione $F 2$.

foFl: frequenza critica per il raggio ordinario, per la regione $F 1$. $h^{\prime} F 1$ : altezza virtuale minima di riflessione della regione $F 1$.

$f o E$ : frequenza critica per il raggio ordinario, per la regione $E$. $h^{\prime} E$ : altezza virtuale minima di riflessione della regione $E$.

Nelle colonne indicate con "val " è specificato il numero dei valori numerici sui quali è stato calcolato il valore mediano di frequenza critica e di altezza virtuale riportato nelle due colonne che precedono.

Nelle ultime tre colonne sono riportate le percentuali di apparizione dello strato $E$ sporadico $(E s)$ calcolate come segue:

prima colonna, contraddistinta con " tot " (totale): percentuale delle registrazioni in cui appare lo strato $E$ s rispetio al numero totale di registrazioni atili:

seconda colonna, contraddistinta con $>5$ : percentuale calcolata come sopra, ma prendendo in considerazione solo le registrazioni in cui la frequenza dello strato $E s$ superi il ralore di 5 Mhz:

terza colonna, contraddistinta con $>7$ : percentuale calcolata come sopra, ma prendendo in considerazione solo le registrazioni in cui la frequenza dello strato $E s$ superi il valore di 7 Mhz.

Per il calcolo dei valori mediani si sono adottate le seguenti convenzioni:

1) Se i valori numerici a disposizione sono + o meno, non si calcola il valore mediano.

2) Per la regione $F 2$, se i valori a disposizione sono da 5 a 9, il valore mediano è dato dubbio; analogamente per le percentuali di apparizione dello strato $E s$, la percentuale è data dubbia se il numero delle registrazioni utili è da 5 a 9 . Le regrioni $F l$ ed $E$ invece sono sufficientemente regolari nelle loro caratteristiche perché si possa considerare non dubbio un valore mediano calcolato su almeno 5 valori numerici.

3) In generale un valore mediano è dato dubbio se calcolato su valori numeriei più della metì dei quali sia dubbia.

I valori dati come dubl,i sono caratterizzati dall'esser racchiusi fra parentesi.

Nel periodo marzo 19-19-aprile 1953 sono stati omessi i dati relativi ai mesi di giugno e luglio 1950 ed ai mesi dal marzo allottohre 1952 per mancanza di un sufficiente numero di valori orari: si tratta di periodi nei quali il funzionamento dell'apparato di sondagnio è stato sospeso o ridotto per importanti modifiche circuitali. 
MARZO 1949)

\begin{tabular}{|c|c|c|c|c|c|c|c|c|c|c|c|c|}
\hline \multirow{2}{*}{ जर } & \multicolumn{9}{|c|}{ VALORI MEDIANI MENSILI } & \multicolumn{3}{|c|}{ Perccentliale Es } \\
\hline & foF2 & $h^{\prime} F 2$ & val & forl & h'FI & val & tiE & h'E & val & tot & $>5$ & $>7$ \\
\hline (1)0 & 7,1 & 290 & 12 & & & & & & & 8 & & \\
\hline $\begin{array}{l}01 \\
07\end{array}$ & & & & & & & & & & & & \\
\hline (13 3 & 6,4 & 275 & 12 & & & & & & & 8 & & \\
\hline$(14$ & & & & & & & & & & & & \\
\hline 05 & & & & & & & & & & & & \\
\hline & 3,9 & $2+11$ & 12 & & & & & & & 16 & & \\
\hline 07 & 7,6 & 230 & 12 & $+, 1)$ & 200 & 9 & & & & & & \\
\hline$(1)$ & 9,0 & 220 & 12 & 4,4 & 200 & 12 & 3.11 & 1001 & 7 & 16 & 8 & \\
\hline 10 & (89) & $1030)$ & $x$ & $=0$ & 700 & 8 & & & & 12 & & \\
\hline 11 & & & & & & & & & & & & \\
\hline 12 & 10,2 & 230 & 13 & 4,5 & 200 & 11 & & & & & & \\
\hline 13 & & & & & & & & & & & & \\
\hline 15 & 10,2 & 230 & 12 &,+ 6 & 200 & 10 & & & & & & \\
\hline 16 & & & & & & & & & & & & \\
\hline 17 & & & & & & & & & & & & \\
\hline 18 & 10,0 & 220 & 10) & & & & & & & 27 & & \\
\hline 19) & $(9,0)$ & $(2201)$ & 9 & & & & & & & 22 & & \\
\hline 20 & 8,3 & 2301 & 12 & & & & & & & 25 & & \\
\hline 21 & & & & & & & & & & & & \\
\hline$\frac{2}{23}$ & 7,7 & 250 & 13 & & & & & & & 7 & & \\
\hline
\end{tabular}

APRILE 1949

\begin{tabular}{|c|c|c|c|c|c|c|c|c|c|c|c|c|}
\hline \multirow{2}{*}{ OKA } & \multicolumn{9}{|c|}{ VALORI MEDIANI MENSILI } & \multicolumn{3}{|c|}{ JERCENTLALE Es } \\
\hline & foF2 & $h^{\prime} F 2$ & bal & $\mathrm{foF}$ & h'F1 & val & foE & $h^{\prime} E$ & val & tot & $>5$ & $>7$ \\
\hline$(10)$ & 7.8 & 275 & 26 & & & & & & & 11 & & \\
\hline $\begin{array}{l}01 \\
0 ?\end{array}$ & & & & & & & & & & & & \\
\hline 0.3 & 7,0 & 270 & 27 & & & & & & & 7 & & \\
\hline (1) & & & & & & & & & & & & \\
\hline $\begin{array}{l}05 \\
06\end{array}$ & $7+$ & 2311 & 26 & +2 & 205 & 20 & 2,5 & 100 & 20 & & & \\
\hline (1) & 8,2 & 220 & 24 & 4,3 & 200 & 24 & 2.8 & 100 & 23 & 8 & & \\
\hline 08 & 8,5 & 220 & 25 & 4,5 & 200 & 24 & 3,2 & 100 & 22 & + & & \\
\hline 10 & 90 & 260 & 23 & +7 & 200 & 12 & $3 .+$ & 100 & 9) & 16 & 8 & \\
\hline 11 & & & & & & & & & & & & \\
\hline 12 & 9,1 & $2 \%$ & 23 & 5.2 & 200 & 17 & 4,11 & 1001 & 7 & & & \\
\hline $\begin{array}{l}13 \\
1+\end{array}$ & & & & & & & & & & & & \\
\hline 15 & 9,5 & 3011 & 24 &,+ 5 & 200 & 21 & 3,3 & 100 & 12 & 16 & 12 & \\
\hline 16 & & & & & & & & & & & & \\
\hline 17 & & & & & & & & & & & & \\
\hline 18 & 9,0 & $2+0$ & 27 & +.5 & 200 & 14 & 2,5 & 100 & 18 & 37 & 11 & \\
\hline 19 & 8,7 & 2311 & 26 & & & & & & & 58 & 8 & \\
\hline 20 & 8,0 & 220 & 26 & & & & & & & 38 & 4 & \\
\hline 21 & & & & & & & & & & & & \\
\hline 22 & 8,0 & 260 & 25 & & & & & & & 8 & & \\
\hline 23 & & & & & & & & & & & & \\
\hline
\end{tabular}


MAGGIO 1949

\begin{tabular}{|c|c|c|c|c|c|c|c|c|c|c|c|c|}
\hline \multirow{2}{*}{ ORA } & \multicolumn{9}{|c|}{ MEDIA N I } & \multicolumn{3}{|c|}{ Prircentuaie Es } \\
\hline & foF? & $h^{\prime} F 2$ & val & foFl & $h^{\prime} F 1$ & val & foE & $h^{\prime} E$ & val & tot & $>5$ & $>7$ \\
\hline 00 & 7,3 & 280 & 24 & & & & & & & 21 & 4 & \\
\hline 01 & & & & & & & & & & & & \\
\hline 02 & & & & & & & & & & & & \\
\hline 03 & 7,0 & 270 & 26 & & & & & & & 31 & 4 & t \\
\hline 04 & 6,6 & 280 & 25 & & & & & & & 32 & & \\
\hline 05 & 6,3 & 260 & 26 & & & & & & & 23 & 4 & \\
\hline 06 & 6,9 & 260 & 22 & 4,0 & 220 & 20 & 2.7 & 110 & 15 & ) & & \\
\hline 07 & 7.5 & 260 & 21 & 4,3 & 220 & 18 & 2,8 & 110 & 12 & 9 & & \\
\hline 08 & 8,0 & 260 & 13 & 4,6 & 220 & 11 & & & & 28 & 7 & \\
\hline 09 & & & & & & & & & & & & \\
\hline 10 & $(8,0)$ & $(260)$ & 9 & 5,0 & 220 & 6 & & & & 33 & 16 & \\
\hline 11 & & & & & & & & & & & & \\
\hline 12 & $(8,0)$ & $(290)$ & 7 & 5,4 & 220 & 6 & $(3,2)$ & (110) & 1 & 10) & 10 & \\
\hline 13 & & & & & & & & & & & & \\
\hline 14 & 80 & 300 & 13 & 50 & 230 & 11 & (1) & $(105)$ & 2 & 8 & 8 & \\
\hline $\begin{array}{l}15 \\
16\end{array}$ & 8,0 & 300 & 17 & $\begin{array}{l}4,0 \\
4,8\end{array}$ & 220 & 15 & 3,7 & 105 & $\overline{7}$ & 28 & 16 & 16 \\
\hline 17 & 7,8 & 270 & 17 & 4,5 & 200 & 12 & 3,3 & 110 & 5 & 47 & 37 & 26 \\
\hline 18 & 8,0 & 260 & 26 & 4,3 & 220 & 17 & 2,8 & 105 & 14 & 48 & 15 & 11 \\
\hline 19 & 7,7 & 250 & 26 & & & & & & & 56 & 22 & 7 \\
\hline 20 & 7,3 & 275 & 23 & & & & & & & 44 & 12 & \\
\hline 21 & & & & & & & & & & & & \\
\hline 22 & 7,3 & 260 & 24 & & & & & & & 54 & 21 & 8 \\
\hline 23 & & & & & & & & & & & & \\
\hline
\end{tabular}

GIUGNO 1949

\begin{tabular}{|c|c|c|c|c|c|c|c|c|c|c|c|c|}
\hline \multirow{2}{*}{ ORA } & \multicolumn{9}{|c|}{ VALORI MEDIANI MENSILI } & \multicolumn{3}{|c|}{ Pl:rentuala: Es } \\
\hline & foF2 & $h^{\prime} F 2$ & val & foF1 & $h^{\prime} F 1$ & val & foE & $h^{\prime} E$ & val & tot & $>5$ & $>7$ \\
\hline 00 & 7,3 & 280 & 27 & & & & & & & 50 & 25 & 11 \\
\hline $\begin{array}{l}01 \\
02\end{array}$ & & & & & & & & & & & & \\
\hline 03 & 6,7 & 270 & 28 & & & & & & & 48 & 21 & 7 \\
\hline 04 & 6,5 & 270 & 29 & & & & & & & 28 & 14 & 7 \\
\hline 05 & 6,8 & 270 & 29 & 3,4 & 240 & 14 & 2,4 & 110 & 9 & 28 & 20 & \\
\hline 06 & 7,5 & 280 & 29 & 4,2 & 220 & 25 & 2,7 & 110 & 26 & 56 & 28 & 7 \\
\hline 07 & 7,8 & 270 & 30 & 4,6 & 210 & 20 & 2,9 & 100 & 23 & 73 & +0 & 7 \\
\hline 08 & 7,4 & 270 & 25 & 4,8 & 210 & 18 & 3,3 & 100 & 10 & 63 & 44 & 15 \\
\hline $\begin{array}{l}09 \\
10\end{array}$ & 74 & $32=$ & & & & 17 & & & & 50 & 13 & 11 \\
\hline $\begin{array}{l}10 \\
11\end{array}$ & 1,4 & 320 & 22 & 3,3 & 210 & 17 & - & - & & 30 & 43 & 11 \\
\hline 12 & 8,0 & 310 & 24 & 5,4 & 200 & 18 & - & - & & 57 & 48 & 5 \\
\hline $\begin{array}{l}13 \\
14\end{array}$ & & & & & & & & & & & & \\
\hline 15 & & 300 & & & & & & & & & & \\
\hline 16 & 7,8 & 300 & 28 & 5,2 & 300 & 22 & $\overline{30}$ & $1 \overline{-}$ & & $\begin{array}{l}45 \\
30\end{array}$ & 30 & $\stackrel{f}{7}$ \\
\hline 17 & 7,5 & 295 & 29 & 4,8 & 220 & 25 & 2,8 & 100 & 12 & 55 & 31 & 7 \\
\hline 18 & 7,7 & 270 & 30 & 4,4 & 220 & 25 & 2,8 & 100 & 20 & 60 & 37 & 3 \\
\hline 19 & 7,7 & 255 & 27 & 3,9 & 210 & 6 & 2,8 & 100 & 7 & 59 & 35 & 7 \\
\hline 20 & 7,7 & 250 & 30 & & & & & & & 73 & 47 & 17 \\
\hline 21 & & & & & & & & & & & & \\
\hline 22 & 7,3 & 265 & 27 & & & & & & & 74 & 48 & 15 \\
\hline 23 & & & & & & & & & & & & \\
\hline
\end{tabular}


LUGLIO 1949

\begin{tabular}{|c|c|c|c|c|c|c|c|c|c|c|c|c|}
\hline \multirow{2}{*}{ ORA } & \multicolumn{9}{|c|}{ VALORI MEDIANI MENSILI } & \multicolumn{3}{|c|}{ Percentuale Es } \\
\hline & foF2 & $h^{\prime} F 2$ & $\mathrm{val}$ & foF1 & $h^{\prime} F 1$ & val & foE & $h^{\prime} E$ & val & tot & $>5$ & $>7$ \\
\hline 00 & 7,0 & 260 & 27 & & & & & & & 67 & 18 & \\
\hline $\begin{array}{l}01 \\
0 ?\end{array}$ & & & & & & & & & & & & \\
\hline 03 & 6,1 & 245 & 27 & & & & & & & 37 & t & \\
\hline 04 & 6,0 & 230) & 27 & & & & & & & 33 & 4 & \\
\hline 05 & 5,6 & 250 & 26 & 4,0 & 220 & 11 & 2,9 & 100 & 8 & 31 & & \\
\hline 06 & 7,1 & 260 & 27 & 4,2 & 220 & 25 & 3,2 & 100 & 21 & +1 & 4 & \\
\hline 07 & 7,1 & 250 & 25 & 4,5 & 210) & 22 & 3,5 & 100 & 19 & 50 & 8 & 4 \\
\hline $\begin{array}{l}08 \\
09\end{array}$ & 7,0 & 260 & 23 & 4,3 & 200 & 19 & 3,6 & 100 & 16 & 26 & 4 & \\
\hline 10 & 7,6 & 300 & 23 & 5,0 & 220 & 18 & 3,6 & 100 & 6 & 48 & 13 & \\
\hline $\begin{array}{l}11 \\
12\end{array}$ & 7,7 & 300 & 23 & 5,0 & 220 & 17 & - & - & & 16 & 20 & 10 \\
\hline 13 & & & & & & & - & - & & +6 & 29 & 12 \\
\hline 14 & & & & & & & & & & & & \\
\hline 15 & 7,6 & 300 & 25 & 5,0 & 220 & 22 & 3,6 & 100 & 10 & 38 & 31 & 11 \\
\hline 16 & 7,5 & 300 & 26 & 4,8 & 210 & 22 & 3,5 & 100 & 13 & 38 & 31 & 15 \\
\hline 17 & 7,6 & 280 & 26 & 4,7 & 220 & 24 & 3,5 & 100 & 18 & 38 & 23 & 8 \\
\hline 18 & 7,6 & 260 & 24 & 4,2 & 220 & 17 & 3,2 & 100 & 15 & +4 & 20 & 8 \\
\hline 19 & 7,8 & 230 & 26 & 4,2 & 210 & 7 & 2,9 & 100 & 6 & 60 & 18 & 7 \\
\hline 20 & 7,7 & 220 & 26 & & & & & & & 63 & 26 & 15 \\
\hline 21 & & & & & & & & & & & & \\
\hline $\begin{array}{l}22 \\
23\end{array}$ & 7,3 & 230 & 26 & & & & & & & 70 & 31 & 8 \\
\hline
\end{tabular}

AGOSTO 1949

\begin{tabular}{|c|c|c|c|c|c|c|c|c|c|c|c|c|}
\hline \multirow{2}{*}{ ORA } & \multicolumn{9}{|c|}{ VALORI MEDIANI } & \multicolumn{3}{|c|}{ Percentuale Es } \\
\hline & foF2 & $h^{\prime} F 2$ & val & foF1 & $h^{\prime} \mathrm{F} 1$ & val & foE & $h^{\prime} E$ & val & tot & $>5$ & $>7$ \\
\hline 00 & 6,2 & 270 & 19 & & & & & & & 26 & 5 & \\
\hline $\begin{array}{l}01 \\
02\end{array}$ & & & & & & & & & & & & \\
\hline 0.3 & 5,8 & 275 & 19 & & & & & & & +2 & 5 & \\
\hline 04 & 5,6 & 250 & 19 & & & & & & & 26 & & \\
\hline 05 & 5,3 & 250 & 18 & & & & & & & 22 & & \\
\hline 06 & 6,0 & 240 & 18 & 3,8 & 210 & 13 & 2,4 & 100 & 8 & 16 & & \\
\hline 07 & 7,3 & 230 & 17 & 4,2 & 200 & 13 & 2,6 & 100 & 13 & 35 & & \\
\hline $\begin{array}{l}08 \\
09\end{array}$ & 7,0 & 250 & 15 & 4,3 & 200 & 13 & 3,0 & 100 & 13 & 56 & 12 & 12 \\
\hline $\begin{array}{l}10 \\
11\end{array}$ & 7,8 & 250 & 16 & 4,8 & 200 & 13 & 3,2 & 200 & 5 & 30 & 6 & \\
\hline 12 & 8,2 & 285 & 16 & 5,2 & 200 & 14 & - & - & & 35 & 35 & 12 \\
\hline $\begin{array}{l}1.3 \\
14\end{array}$ & & & & & & & & & & & & \\
\hline 15 & 8,1 & 290 & 18 & 4,7 & 200 & 18 & 3,4 & 100 & 8 & 16 & 5 & 5 \\
\hline 16 & 8,5 & 270 & 19 & 4,7 & 200 & 16 & 3,3 & 100 & 5 & 21 & 10 & 5 \\
\hline 17 & 8,0 & 250 & 20 & 4,5 & 210 & 12 & 2,8 & 100 & 11 & 30 & 25 & 5 \\
\hline 18 & 7,8 & 250 & 20 & 3,9 & 210 & 10 & 2,5 & 100 & 13 & 28 & & \\
\hline 19 & 7,6 & 220 & 16 & & & & & & & 35 & 15 & 10 \\
\hline 20 & 7,4 & 220 & 16 & & & & & & & 37 & 12 & \\
\hline $\begin{array}{l}21 \\
22\end{array}$ & 6,7 & 250 & 19 & & & & & & & 60 & 10 & 5 \\
\hline 23 & & & & & & & & & & & & \\
\hline
\end{tabular}


PIERO DOMIYICI

SETTEMBRE 1949

\begin{tabular}{|c|c|c|c|c|c|c|c|c|c|c|c|c|}
\hline \multirow{2}{*}{ ORA } & \multicolumn{9}{|c|}{ VALORL MEDIANI MENSILI } & \multicolumn{3}{|c|}{ Percientualie Es } \\
\hline & for 2 & $h^{\prime} F 2$ & val & toF 1 & $h^{\prime} F 1$ & c.al & foE & $h^{\prime} E$ & vil & tot & $\because 5$ & $>7$ \\
\hline 00 & 6,0 & 270 & $2 k$ & & & & & & & 21 & t & \\
\hline $\begin{array}{l}01 \\
02\end{array}$ & & & & & & & & & & & & \\
\hline 03 & 5,3 & 260 & 28 & & & & & & & 10 & 4 & \\
\hline 04 & 5,2 & 260 & 28 & & & & & & & 10) & 4 & \\
\hline 05 & $\overline{5}, 1$ & 250 & 28 & & & & & & & 7 & & \\
\hline 06 & 5,6 & 230 & 28 & 3.4 & 220 & 6 & $2,1)$ & 100 & 3 & 7 & \pm & 4 \\
\hline 07 & 7,1 & 220 & 26 & 3,7 & 220 & 9 & 2.5 & 100 & 19 & 7 & 7 & \\
\hline 08 & $7, \overline{3}$ & 230 & 21 & $+, 1)$ & 200 & 11 & 2,8 & 100 & 19 & 17 & 4 & \\
\hline $\begin{array}{l}09 \\
10\end{array}$ & 7,6 & $2+5$ & 20 & 4.3 & 200 & 18 & 3,0 & 100 & $1 \mathrm{~s}$ & $y$ & & \\
\hline 11 & & & & & & & & & & & & \\
\hline 12 & 8,0 & 250 & 23 & 5,0 & 200 & 21 & 3.3 & 100 & 14 & 15 & 8 & 4 \\
\hline 13 & & & & & & & & & & & & \\
\hline 14 & & & & & & & & & & & & \\
\hline 15 & 7,8 & 250 & 25 & 4,6 & 205 & 20 & 3.1 & $10 n$ & 22 & 20 & 8 & 4 \\
\hline 16 & 7,7 & 250 & 25 & 4.5 & 210 & 18 & 3,2 & 100 & 22 & 11 & \pm & \\
\hline 17 & 7,8 & 230 & 25 & 4,3 & 220 & 12 & 2,5 & 100 & 20 & 30) & 15 & \\
\hline 18 & $7, \overline{5}$ & 220 & 22 & & & & 2,0 & 100 & 6 & 30 & 15 & \\
\hline 19 & 7,0 & 215 & 23 & & & & & & & 4 & 12 & \\
\hline 20 & & & & & & & & & & 46 & 15 & \\
\hline $\begin{array}{l}21 \\
27\end{array}$ & 65 & $7+0$ & & & & & & & & 48 & + & \\
\hline 23 & 0,3 & -40 & -1 & & & & & & & & & \\
\hline
\end{tabular}

OTTOBRE 1949

\begin{tabular}{|c|c|c|c|c|c|c|c|c|c|c|c|c|}
\hline \multirow{2}{*}{ ORA } & \multicolumn{9}{|c|}{ VALORI MEDIANI MENSILI } & \multicolumn{3}{|c|}{ Percentuale Es } \\
\hline & foF2 & h'F2 & val & foFl & h'F1 & val & foE & $h^{\prime} E$ & al & tot & $>5$ & $>7$ \\
\hline 00 & 5,8 & 280 & 28 & & & & & & & $1+$ & & \\
\hline $\begin{array}{l}01 \\
02\end{array}$ & & & & & & & & & & & & \\
\hline 03 & 5,0 & 300 & 29 & & & & & & & $\overrightarrow{7}$ & & \\
\hline 04 & 5,0 & 295 & 29 & & & & & & & 7 & & \\
\hline 05 & 4,8 & 265 & 28 & & & & & & & & & \\
\hline 06 & 4,8 & 260 & 28 & & & & & & & & & \\
\hline 07 & 7,6 & 220 & 28 & & & & 2.6 & 105 & 12 & 4 & & \\
\hline 08 & 8,1 & 220) & 28 & & & & 3,1 & 100 & 11 & 7 & & \\
\hline 09 & & & & & & & & & & & & \\
\hline 10 & 8,2 & 220 & 27 & & & & 3.5 & 100) & 8 & 18 & 11 & \\
\hline 12 & 8,3 & 220 & 24 & & & & - & - & & 36 & 21 & 7 \\
\hline 13 & & & & & & & & & & & & \\
\hline 14 & & 200 & 28 & & & & 35 & 100 & 13 & 24 & $1+$ & \\
\hline 16 & $\begin{array}{l}8,3 \\
8,4\end{array}$ & 220 & $\begin{array}{l}-88 \\
28\end{array}$ & & & & $\begin{array}{l}3,5 \\
3,2\end{array}$ & 100 & 12 & 31 & 14 & \\
\hline 17 & 8,2 & 220) & 29 & & & & 2,7 & 100 & 10) & 24 & 11 & 4 \\
\hline 18 & 7,9 & 220 & 27 & & & & & & & 55 & 11 & 4 \\
\hline 19 & 7,1 & 220) & 23 & & & & & & & 45 & 14 & 4 \\
\hline 20 & 6,8 & 240 & 23 & & & & & & & 35 & 14 & 4 \\
\hline 21 & & & & & & & & & & & & \\
\hline 22 & 6,0 & 260 & 27 & & & & & & & 38 & $\gamma$ & t \\
\hline
\end{tabular}


VALOHI MEUIANI MENSILI MELLE CARATTERISTICHE IONOSFERICHF I FOMA

NOVEMBRE 1949

\begin{tabular}{|c|c|c|c|c|c|c|c|c|c|c|c|c|}
\hline \multirow{2}{*}{ ORA } & \multicolumn{9}{|c|}{ VALORI MEDIANI } & \multicolumn{3}{|c|}{ Percentuale Es } \\
\hline & toF2 & $h^{\prime} F 2$ & val & foF1 & $h^{\prime} F 1$ & val & foE & $h^{\prime} E$ & val & tot & $>5$ & $>7$ \\
\hline 00 & 4,7 & 260 & 24 & & & & & & & 8 & & \\
\hline 01 & & & & & & & & & & & & \\
\hline 02 & 13 & 964 & 71 & & & & & & & & & \\
\hline 04 & 4,1 & 265 & 24 & & & & & & & & & \\
\hline 05 &,+ 0 & 240 & 24 & & & & & & & & & \\
\hline 06 & 3,9 & 250 & 24 & & & & & & & & & \\
\hline (07 & 6,0 & 220 & 23 & & & & & & & & & \\
\hline 118 & 7.7 & 200 & 19 & & & & & & & & & \\
\hline (1) & & & & & & & & & & & & \\
\hline 10 & $(8,5)$ & 2.30 & sis & 5,0 & 200 & 5 & 3,5 & 100 & 7 & 36 & 9 & \\
\hline 11 & & & & & & & & & & & & \\
\hline 12 & $(8,9) !$ & $(2.30)$ & $\dot{x}$ & 5,0 & 200 & 5 & 3,7 & 100 & 6 & +5 & 18 & 9 \\
\hline $\begin{array}{l}13 \\
14\end{array}$ & & & & & & & & & & & & \\
\hline 15 & 8,5 & 230 & 14 & 5,0 & 200 & 8 & 3,5 & 105 & 6 & 6 & & \\
\hline 16 & 7,7 & 220 & 20 & 4,4 & 200 & 8 & 3,4 & 100 & 5 & 10 & & \\
\hline 17 & 7,4 & 200 & 19 & & & & & & & 21 & & \\
\hline 18 & 6,8 & 200 & 13 & & & & & & & 14 & & \\
\hline 19 & 6,0 & 220 & 16 & & & & & & & 12 & & \\
\hline 20 & 5,8 & 220 & 19 & & & & & & & & & \\
\hline 21 & & & & & & & & & & & & \\
\hline 22 & 5,3 & 245 & 21 & & & & & & & 10 & & \\
\hline 23 & & & & & & & & & & & & \\
\hline
\end{tabular}

DICEMBRE 1949

\begin{tabular}{|c|c|c|c|c|c|c|c|c|c|c|c|c|}
\hline \multirow{2}{*}{ ORA } & \multicolumn{9}{|c|}{ YALOKI MEDIANI MENSILI } & \multicolumn{3}{|c|}{ Percentuate Es } \\
\hline & foF2 & h'F2 & val & forl & $h^{\prime} \mathrm{F} 1$ & val & $f_{0} E$ & $h^{\prime} E$ & val & tot & $>5$ & $>7$ \\
\hline$(60$ & 4,0 & 250 & 12 & & & & & & & & & \\
\hline (1) 1 & & & & & & & & & & & & \\
\hline 02 & +2 & $300)$ & 11 & & & & & & & 17 & & \\
\hline 0.4 & 4,2 & 255 & 12 & & & & & & & 17 & & \\
\hline 05 & 4.1 & 250 & 12 & & & & & & & 8 & & \\
\hline 06 & 4.0 & 240 & 12 & & & & & & & & & \\
\hline 07 & 4,2 & 220) & 11 & & & & & & & & & \\
\hline 08 & 7,0 & $2(x)$ & 11 & & & & & & & 18 & & \\
\hline 09 & & & & & & & & & & & & \\
\hline 10 & 8,0 & 210 & 10 & & & & & & & & & \\
\hline $\begin{array}{l}11 \\
17\end{array}$ & & & 10 & 50 & 20 & 6 & & & & 9 & & \\
\hline 13 & & & & & & & & & & & & \\
\hline 14 & & & & & & & & & & & & \\
\hline 15 & 8,2 & 200 & 11 & 4,7 & 200 & 5 & & & & 9 & & \\
\hline 16 & 7,8 & 200 & 11 & & & & & & & 9 & & \\
\hline 17 & 7,5 & 200 & 11 & & & & & & & 18 & & \\
\hline 18 & 6,7 & 200 & 11 & & & & & & & 54 & 9 & \\
\hline 19 & 5,7 & 210) & 12 & & & & & & & 17 & & \\
\hline 20 & 5,2 & 220) & 1.3 & & & & & & & 15 & & \\
\hline 21 & & & & & & & & & & & & \\
\hline 22 & $4,1)$ & 240 & 13 & & & & & & & 8 & & \\
\hline 23 & & & & & & & & & & & & \\
\hline
\end{tabular}


GENNAIO 1950

\begin{tabular}{|c|c|c|c|c|c|c|c|c|c|c|c|c|}
\hline \multirow{2}{*}{ ORA } & \multicolumn{9}{|c|}{ VALORI MEDIANI MENSILI } & \multicolumn{3}{|c|}{ Percentuale Es } \\
\hline & foF2 & h'F2 & val & for1 & $h^{\prime} F 1$ & val & foE & $h^{\prime} E$ & vil & tot & $>5$ & $>7$ \\
\hline 00 & 4,2 & 260 & 25 & & & & & & & 16 & 8 & \\
\hline 01 & & & & & & & & & & & & \\
\hline 02 & & & & & & & & & & & & \\
\hline 03 & 4,4 & 260 & 25 & & & & & & & & & \\
\hline 04 & 4,4 & 250 & 25 & & & & & & & & & \\
\hline 05 & 4,2 & 240 & 24 & & & & & & & & & \\
\hline 06 & 3,9 & 240 & 24 & & & & & & & & & \\
\hline 07 & 4,2 & 230 & 26 & & & & & & & & & \\
\hline 08 & 8,0 & 200 & 28 & & & & & & & & & \\
\hline 09 & & & & & & & & & & & & \\
\hline 10 & 10,0 & 210 & 18 & 4,6 & 200 & 9 & 3,3 & 110 & 11 & 7 & 4 & 4 \\
\hline $\begin{array}{l}11 \\
12\end{array}$ & 11,0 & 210 & 16 & 5,0 & 200 & 14 & 3,5 & 100 & 10 & 4 & 4 & \\
\hline 13 & & & & & & & & & & & & \\
\hline 14 & & & & & & & & & & & & \\
\hline 15 & 11,0 & 210 & 24 & 4,4 & 205 & 12 & 3,2 & 100 & 9 & & & \\
\hline 16 & 11,0 & 200 & 25 & & & & & & & 4 & & \\
\hline 17 & 9,5 & 200 & 27 & & & & & & & 7 & & \\
\hline 18 & 7,5 & 205 & 29 & & & & & & & 10 & 3 & \\
\hline 19 & 6,1 & 210 & 29 & & & & & & & 14 & & \\
\hline 20 & 5,1 & 210 & 29 & & & & & & & 10 & & \\
\hline 21 & & & & & & & & & & & & \\
\hline 22 & 4,3 & 260 & 26 & & & & & & & 4 & & \\
\hline 23 & & & & & & & & & & & & \\
\hline
\end{tabular}

FEBBRAIO 1950

\begin{tabular}{|c|c|c|c|c|c|c|c|c|c|c|c|c|}
\hline \multirow{2}{*}{ ORA } & \multicolumn{9}{|c|}{ VALORI MEDIANI MENSILI } & \multicolumn{3}{|c|}{ Percentuale Es } \\
\hline & foF2 & $h^{\prime} \mathrm{F} 2$ & val & for1 & h'F1 & val & foE & $h^{\prime} E$ & val & tot & $>5$ & $=7$ \\
\hline 00 & 4,2 & 260 & 18 & & & & & & & & & \\
\hline 01 & & & & & & & & & & & & \\
\hline 02 & & & & & & & & & & & & \\
\hline 03 & 4,1 & 250 & 16 & & & & & & & & & \\
\hline 04 & 4,1 & 255 & 14 & & & & & & & & & \\
\hline 05 & 3,9 & 245 & 16 & & & & & & & & & \\
\hline 06 & 3,0 & 235 & 16 & & & & & & & & & \\
\hline 07 & 4,0 & 230 & 16 & & & & & & & 7 & & \\
\hline 08 & 7,5 & 200 & 18 & & & & & & & & & \\
\hline 09 & & & & & & & & & & & & \\
\hline 10 & $(10,0)$ & 220 & 12 & 4,3 & 200 & 5 & & & & & & \\
\hline 11 & & & & & & & & & & & & \\
\hline 12 & $(10,5)$ & 230 & 13 & 4,9 & 200 & 8 & & & & & & \\
\hline 13 & & & & & & & & & & & & \\
\hline $\begin{array}{l}17 \\
15\end{array}$ & 11,0 & 210 & 17 & 4,2 & 200 & 6 & & & & 6 & 6 & \\
\hline 16 & 9,5 & 210 & 16 & & & & & & & 12 & 6 & \\
\hline 17 & 9,0 & 200 & 17 & & & & & & & 17 & 12 & \\
\hline 18 & 7,5 & 205 & 16 & & & & & & & & & \\
\hline 19 & 6,3 & 210 & 16 & & & & & & & 12 & & \\
\hline 20 & 5,4 & 220 & 17 & & & & & & & 6 & & \\
\hline 21 & & & & & & & & & & & & \\
\hline 22 & 4,0 & 250 & 17 & & & & & & & & & \\
\hline 23 & & & & & & & & & & & & \\
\hline
\end{tabular}


YaloR I MEdiani MENSILI deLle Caratteristiche ionosferiche ～RoM.

MARZO 1950

\begin{tabular}{|c|c|c|c|c|c|c|c|c|c|c|c|c|}
\hline \multirow{2}{*}{ ORA } & \multicolumn{9}{|c|}{ VALORI MEDIANI MENSILI } & \multicolumn{3}{|c|}{ Percentuale Es } \\
\hline & foF2 & h'F2 & val & foF 1 & $h^{\prime} F 1$ & val & foE & $h^{\prime} \mathbf{E}$ & val & tot & $>5$ & $>7$ \\
\hline 00 & 4,8 & 260 & 13 & & & & & & & 7 & & \\
\hline 01 & & & & & & & & & & & & \\
\hline 02 & & & & & & & & & & & & \\
\hline $\begin{array}{l}03 \\
04\end{array}$ & $\begin{array}{l}4,5 \\
4,0\end{array}$ & $\begin{array}{l}260 \\
260\end{array}$ & $\begin{array}{l}13 \\
13\end{array}$ & & & & & & & & & \\
\hline 05 & 3,3 & 260 & 13 & & & & & & & & & \\
\hline 06 & 4,8 & 250 & 13 & & & & & & & & & \\
\hline 07 & 6,0 & 210 & 13 & & & & & & & & & \\
\hline 08 & 7,3 & 220 & 13 & 3,0 & 200 & 7 & & & & 7 & & \\
\hline 09 & & & & & & & & & & & & \\
\hline $\begin{array}{l}10 \\
11\end{array}$ & $(8,3)$ & 230 & 10 & 3,7 & 200 & 7 & & & & & & \\
\hline 12 & $(9,3)$ & 240 & 10 & 4,5 & 200 & 9 & & & & & & \\
\hline 13 & & & & & & & & & & & & \\
\hline 14 & & & & & & & & & & & & \\
\hline 15 & $(9,0)$ & 240 & 13 & 4,2 & 200 & 13 & & & & & & \\
\hline 16 & $(8,5)$ & 230 & 11 & 3,8 & 200 & 9 & & & & & & \\
\hline 17 & 8,0 & 220 & 13 & 3,0 & 200 & 5 & & & & 15 & 7 & \\
\hline 18 & 8,5 & 210 & 13 & & & & & & & 7 & & \\
\hline 19 & 8,0 & 205 & 13 & & & & & & & 15 & & \\
\hline 20 & 6,7 & 210 & 14 & & & & & & & 14 & & \\
\hline 21 & & & & & & & & & & 7 & & \\
\hline $\begin{array}{l}22 \\
23\end{array}$ & 5,8 & 240 & 14 & & & & & & & 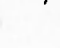 & & \\
\hline
\end{tabular}

APRILE 1950

\begin{tabular}{|c|c|c|c|c|c|c|c|c|c|c|c|c|}
\hline \multirow{2}{*}{ ORA } & \multicolumn{9}{|c|}{ VALOR I MEDIA N I } & \multicolumn{3}{|c|}{ Percentuale Es } \\
\hline & foF2 & $h^{\prime} \mathbf{F} 2$ & val & foF1 & h'F1 & val & foE & $h^{\prime} E$ & val & tot & $>5$ & $>7$ \\
\hline 00 & 6.0 & 270 & 23 & & & & & & & 9 & 4 & \\
\hline 01 & & & & & & & & & & & & \\
\hline 03 & 6,0 & 255 & 23 & & & & & & & 4 & & \\
\hline 04 & 5,0 & 250 & 22 & & & & & & & 4 & & \\
\hline 05 & 4,8 & 260 & 23 & & & & & & & 9 & & \\
\hline 06 & 5,3 & 220 & 23 & & & & & & & 4 & & \\
\hline 07 & 6,2 & 225 & 24 & 3,2 & 200 & 17 & 2,5 & 100 & 10 & 4 & & \\
\hline 08 & 7,3 & 230 & 24 & 3,7 & 200 & 18 & 2,7 & 100 & 11 & 4 & & \\
\hline 09 & & & & & & 16 & & & & 9 & 9 & \\
\hline 10 & 9,0 & 250 & 21 & 4,2 & 200 & 16 & & & & 9 & 9 & \\
\hline 12 & 9,4 & 260 & 19 & 5,0 & 200 & 17 & & & & 9 & & \\
\hline 13 & & & & & & & & & & & & \\
\hline 14 & & & & & e & & & & & & & \\
\hline 15 & 9,5 & 250 & 19 & 4,3 & 200 & 20 & 2,9 & 100 & 9 & 4 & & \\
\hline 16 & 9,0 & 250 & 23 & 4,0 & 205 & 19 & 2,8 & 100 & 13 & 17 & 4 & \\
\hline 17 & $8, \overrightarrow{5}$ & 240 & 22 & 3,9 & 210 & 13 & 2,7 & 100 & 13 & 41 & 4 & \\
\hline 18 & 8,2 & 220 & 22 & & & & 2,5 & 100 & 6 & 59 & 4 & \\
\hline 19 & 8,0 & 220 & 22 & & & & & & & 45 & 4 & \\
\hline 20 & 7,5 & 230 & 22 & & & & & & & 41 & & \\
\hline 21 & & & & & & & & & & & & \\
\hline 22 & 6,2 & 250 & 23 & & & & & & & 30 & $t$ & \\
\hline 23 & & & & & & & & & & & & \\
\hline
\end{tabular}


MAGGIO 1950

\begin{tabular}{|c|c|c|c|c|c|c|c|c|c|c|c|c|}
\hline \multirow{2}{*}{ ORA } & \multicolumn{9}{|c|}{ MENSILI } & \multicolumn{3}{|c|}{ Percentuale Es } \\
\hline & foF2 & h'F2 & val & for 1 & h'F1 & val & for $E$ & h'E & val & tot & $>5$ & $>7$ \\
\hline 00 & 6,3 & 290 & 25 & & & & & & & 45 & 4 & \\
\hline $\begin{array}{l}01 \\
07\end{array}$ & & & & & & & & & & & & \\
\hline 03 & 5,9 & 270 & 27 & & & & & & & 60 & & \\
\hline 04 & 5,2 & 280 & 27 & & & & & & & 33 & 4 & \\
\hline 05 & 5,6 & 270 & 27 & & & & & & & 30 & & \\
\hline 06 & 6,1 & 250 & 25 & 3,2 & 210 & 14 & 2.? & 110 & 8 & 22 & & \\
\hline 07 & 6,8 & 250 & 24 & 3,7 & 210 & 17 & 2,3 & 105 & 12 & 45 & 11 & 4 \\
\hline 08 & 6,6 & 250 & 19 & 4,0 & 200 & 6 & & & & 50 & 16 & 4 \\
\hline $\begin{array}{l}09 \\
10\end{array}$ & 78 & 280 & 13 & 16 & $(20)$ & 7 & & & & 25 & $t$ & \\
\hline 11 & & & & & & & & & & & 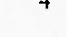 & \\
\hline 12 & $(8,5)$ & 300 & 16 & 5,0 & 200 & 9 & & & & 23 & 15 & 4 \\
\hline 13 & & & & & & & & & & & & \\
\hline $\begin{array}{l}14 \\
15\end{array}$ & 7.8 & 290 & 26 & +6 & 300 & & & & & & & \\
\hline 16 & 7,6 & 280 & 26 & $\begin{array}{l}4,3 \\
4,3\end{array}$ & 200 & 11 & $\begin{array}{l}3,1 \\
3,7\end{array}$ & 100 & 7 & 30 & 15 & 4 \\
\hline 17 & 7,8 & 260 & 25 & 3,9 & 205 & 14 & 2,6 & 100 & 7 & 30 & 15 & \\
\hline 18 & 7,7 & 250 & 25 & & & & & & & 54 & 23 & \\
\hline 19 & 7,3 & 240 & 25 & & & & & & & 54 & 15 & 11 \\
\hline 20 & 7,3 & 230 & 25 & & & & & & & 70 & 11 & \\
\hline 21 & & & & & & & & & & & & \\
\hline $\begin{array}{l}22 \\
23\end{array}$ & 6,4 & 260 & 26 & & & & & & & 52 & 4 & \\
\hline
\end{tabular}

AGOSTO 1950

\begin{tabular}{|c|c|c|c|c|c|c|c|c|c|c|c|c|}
\hline \multirow{2}{*}{ ORA } & \multicolumn{9}{|c|}{ VALORI MEDIANI MENSILI } & \multicolumn{3}{|c|}{ Percentuale Es } \\
\hline & foF2 & $h^{\prime} \mathrm{F} 2$ & val & foF1 & h'F1 & val & foE & $h^{\prime} E$ & nal & tot & $\div 5$ & $>7$ \\
\hline $\begin{array}{l}00 \\
01\end{array}$ & 4,7 & 255 & 26 & & & & & & & 56 & 18 & 7 \\
\hline 02 & & & & & & & & & & & & \\
\hline 03 & 3,7 & 260 & 27 & & & & & & & 48 & 7 & \\
\hline 04 & 3.6 & 250 & 26 & & & & & & & 37 & 7 & 4 \\
\hline 05 & 3,3 & 250 & 26 & & & & & & & 37 & 7 & \\
\hline 06 & 4,4 & 240 & 25 & 2.8 & 210 & 11 & 1,8 & $104)$ & 5 & 42 & 11 & \\
\hline 07 & 5,4 & 240 & 22 & 3,0 & 200 & 18 & 2,0 & 100 & 16 & 46 & 8 & 4 \\
\hline $\begin{array}{l}08 \\
09\end{array}$ & 5,7 & 240 & 20 & 3,6 & 200 & 18 & 2,4 & 100 & 21 & 56 & 20 & $x$ \\
\hline 10 & 6,8 & 265 & 14 & 4,0 & 200 & 13 & 2,8 & 100 & 1) & 40 & 20 & 8 \\
\hline $\begin{array}{l}12 \\
13\end{array}$ & 7,1 & 300 & 16 & 4,5 & 200 & 13 & 2,8 & 100 & 13 & 20 & 12 & \\
\hline 14 & & & & & & & & & & & & \\
\hline 15 & 6,8 & 300 & 23 & 4,0 & 200 & 23 & 2,8 & 100 & 18 & 27 & 4 & 4 \\
\hline 16 & 7,0 & 260 & 25 & 4,0 & 200 & 24 & 2,6 & 100 & 13 & +1 & 26 & 4 \\
\hline 17 & 7,0 & 250 & 27 & 3.5 & 200 & 20 & 2,4 & 100 & 17 & 50 & 21 & 7 \\
\hline 18 & 6,7 & 230 & 28 & 3,0 & 205 & 17 & 2.2 & 100 & 13 & 54 & 11 & 7 \\
\hline 19 & 7,0 & 220 & 26 & & & & & & & 36 & 14 & 7 \\
\hline 20 & 6,5 & 210 & 27 & & & & & & & 55 & 17 & 14 \\
\hline 22 & 5,5 & 225 & 25 & & & & & & & 55 & 24 & 7 \\
\hline 23 & & & & & & & & & & & & \\
\hline
\end{tabular}


VALORI MEUIAN MENSILI DELLE CARATTERISTICHE IONOSFERICHE: 1 RONA

SETTEMBRE 1950

\begin{tabular}{|c|c|c|c|c|c|c|c|c|c|c|c|c|}
\hline \multirow{2}{*}{ ORA } & \multicolumn{9}{|c|}{ VALORI MEDIANI MENSIII } & \multicolumn{3}{|c|}{ Percentuale Es } \\
\hline & toF2 & $h^{\prime} \mathrm{F} 2$ & ral & foF1 & h'F1 & val & toE & $h^{\prime} E$ & val & $\operatorname{tot}$ & $>5$ & $>7$ \\
\hline (0) & 3,3 & 280 & 15 & & & & & & & 33 & 6 & \\
\hline $\begin{array}{l}01 \\
\text { (12 }\end{array}$ & & & & & & & & & & & & \\
\hline 03 & 3,0 & 270 & 16 & & & & & & & 25 & & \\
\hline 114 & 2.9 & 270 & 15 & & & & & & & 18 & & \\
\hline 05 & 2.7 & 265 & 16 & & & & & & & 12 & 6 & \\
\hline 06 & 3.2 & 230 & 14 & & & & & & & 6 & 6 & \\
\hline 07 &,+ 2 & 230 & 16 & 2.5 & $2(1) 0$ & 7 & 2.0 & 100 & 7 & 37 & 6 & \\
\hline 08 & 5.4 & 240 & 14 & 3,3 & $2(10)$ & 9 & 2.4 & 100 & 8 & 21 & & \\
\hline (1)9 & & & & & & & & & & & & \\
\hline 10) & 5,7 & $26(1)$ & 12 & 3,9 & $2(10)$ & 11 & & & & 13 & & \\
\hline 11 & & & & & 8 & & & & & & & \\
\hline 12 & 0.2 & 26,0 & 12 & 4.0 & 200 & 9) & & & & 25 & 12 & 6 \\
\hline $\begin{array}{l}13 \\
14\end{array}$ & & & & & & & & & & & & \\
\hline $\begin{array}{l}14 \\
15\end{array}$ & 6.2 & 250 & 18 & 3.5 & 2110 & 14 & 2.5 & 100 & 3 & 5 & & \\
\hline 16 & 6,4 & 240 & 19 & 3,4 & 200 & 13 & 2,4 & 100 & 7 & 9 & & \\
\hline 17 & 7,0 & 240 & 17 & 3.2 & 2015 & 8 & 2,2 & 100 & 10 & 30 & 5 & \\
\hline 18 & 7.0 & 220 & 19 & & & & & & & 47 & & \\
\hline 19) & 6.3 & 220 & 17 & & & & & & & 39 & 5 & \\
\hline 20 & 6.5 & 220 & 15 & & & & & & & 35 & & \\
\hline 21 & & & & & & & & & & & & \\
\hline 22 & 3,7 & $24(1)$ & 18 & & & & & & & 44 & 5 & \\
\hline 23 & & & & & & & & & & & & \\
\hline
\end{tabular}

OTTOBRE 1950

\begin{tabular}{|c|c|c|c|c|c|c|c|c|c|c|c|c|}
\hline \multirow{2}{*}{ ORA } & \multicolumn{9}{|c|}{ VALOKI MEDIANI MESSILI } & \multicolumn{3}{|c|}{ Percentuaie Es } \\
\hline & foF2 & $h^{\prime} F 2$ & val & iof 1 & h'F1 & ral & to $E$ & $h^{\prime} E$ & ral & tot & $>5$ & $>7$ \\
\hline 00 & 3.2 & 285 & 17 & & & & & & & & & \\
\hline $\begin{array}{l}111 \\
02\end{array}$ & & & & & & & & & & & & \\
\hline 0.3 & 3.0 & 295 & 16 & & & & & & & & & \\
\hline 04 & 3,1 & 275 & 17 & & & & & & & & & \\
\hline 05 & 3,0 & 250 & 16 & & & & & & & & & \\
\hline 06 & 2.7 & $(245)$ & 14 & & & & & & & 6 & & \\
\hline 07 & 4.7 & 215 & 18 & & & & & & & & & \\
\hline 08 & 6.0 & 205 & 19 & 2.5 & 200 & 9 & & & & 16 & & \\
\hline $\begin{array}{l}109 \\
10\end{array}$ & 6.2 & 225 & 15 & 3.3 & $(200)$ & 7 & 3.2 & 110 & 6 & 14 & & \\
\hline 11 & & & & & & & & & & & & \\
\hline 12 & 7.2 & 230 & 15 & 3.8 & (210) & 9 & 3,5 & 100 & 5 & 11) & 10 & 5 \\
\hline $\begin{array}{l}1.3 \\
14\end{array}$ & & & & & & & & & & & & \\
\hline 15 & 7,7 & 225 & 17 & 3.1 & 210 & 8 & 2,4 & 100 & 6 & 6 & & \\
\hline 16 & 7.4 & 220 & 18 & 2.4 & (210) & 7 & & & & 11 & & \\
\hline 17 & 7.3 & 210 & 18 & & & & & & & 16 & & \\
\hline 18 & 6,0 & 210 & 19 & & & & & & & 21 & 5 & \\
\hline 19 & 4,9 & 230 & 20 & & & & & & & 20 & & \\
\hline 20 & 4,7 & 230 & 19 & & & & & & & 21 & & \\
\hline 21 & & & & & & & & & & & & \\
\hline 22 & 3,5 & $(270)$ & 15 & & & & & & & & & \\
\hline 23 & & & & & & & & & & & & \\
\hline
\end{tabular}


NOVEMBRE 1950

\begin{tabular}{|c|c|c|c|c|c|c|c|c|c|c|c|c|}
\hline \multirow{2}{*}{ ORA } & \multicolumn{9}{|c|}{ VALORI MEDIANI MENSILI } & \multicolumn{3}{|c|}{ Percentuale: Es } \\
\hline & foF2 & $h^{\prime} \mathrm{F} 2$ & val & foF1 & h'F1 & val & foE & $h^{\prime} E$ & val & tot & $>5$ & $>7$ \\
\hline 00 & 3,0 & 260 & 22 & & & & & & & 8 & & \\
\hline $\begin{array}{l}01 \\
02\end{array}$ & & & & & & & & & & & & \\
\hline 03 & 2,8 & 270 & 25 & & & & & & & 8 & & \\
\hline 04 & 2,8 & 255 & 25 & & & & & & & 4 & & \\
\hline 05 & 2,9 & 230 & 24 & & & & & & & 4 & & \\
\hline 06 & 2,5 & 230 & 23 & & & & & & & & & \\
\hline 07 & 3,6 & 220 & 25 & & & & & & & 4 & & \\
\hline 08 & 6,4 & 210 & 25 & & & & & & & 8 & 4 & \\
\hline 10 & 7,5 & 215 & 24 & 3,7 & 200 & 17 & 2,8 & 100 & 9 & 12 & 4 & \\
\hline 11 & & & & & & & & & & & & \\
\hline 12 & 7,7 & 220 & 21 & 3,8 & 200 & 15 & 3,0 & 100 & 9 & 30 & 9 & \\
\hline 13 & & & & & & & & & & & & \\
\hline 14 & 77 & & & 40 & 300 & 5 & 27 & 1100 & 5 & & & \\
\hline $\begin{array}{l}15 \\
16\end{array}$ & 7,5 & 210 & 24 & 7,0 & -00 & J & 2,1 & 100 & & 8 & & \\
\hline 17 & 6,0 & 200 & 24 & & & & & & & & & \\
\hline 18 & 4,3 & 215 & 23 & & & & & & & 12 & 4 & \\
\hline 19 & 4,2 & 220 & 22 & & & & & & & 37 & 4 & 4 \\
\hline 20 & 3,2 & 230 & 23 & & & & & & & 13 & & \\
\hline 21 & & & & & & & & & & & & \\
\hline $\begin{array}{l}22 \\
23\end{array}$ & 2,8 & 210 & 18 & & & & & & & 8 & & \\
\hline
\end{tabular}

DICEMBRE 1950

\begin{tabular}{|c|c|c|c|c|c|c|c|c|c|c|c|c|}
\hline \multirow{2}{*}{ ORA } & \multicolumn{9}{|c|}{ VALORI MEDIANI MENSILI } & \multicolumn{3}{|c|}{ Percentuale Es } \\
\hline & foF2 & h'F2 & val & foF1 & $h^{\prime} \mathrm{F} 1$ & val & foE & $h^{\prime} E$ & val & tot & $>5$ & $>7$ \\
\hline 00 & 2,9 & 270 & 12 & & & & & & & 14 & & \\
\hline $\begin{array}{l}01 \\
02\end{array}$ & & & & & & & & & & & & \\
\hline 03 & 2,9 & 265 & 18 & & & & & & & 5 & & \\
\hline 04 & 2,7 & 270 & 17 & & & & & & & & & \\
\hline 05 & 2,7 & 250 & 16 & & & & & & & & & \\
\hline 06 & 2.8 & 245 & 12 & & & & & & & & & \\
\hline 07 & 3,2 & 220 & 14 & & & & & & & 7 & 7 & \\
\hline 08 & 5,2 & 200 & 24 & & & & & & & & & \\
\hline 09 & & & & & & & & & & & & \\
\hline $\begin{array}{l}10 \\
11\end{array}$ & 6,8 & 220 & 22 & 3,1 & 200 & 14 & & & & 9 & 7 & \\
\hline 12 & 7,2 & 200 & 23 & 4,0 & 200 & 12 & & & & 4 & & \\
\hline 13 & & & & & & & & & & & & \\
\hline 14 & & & & & & & & & & & & \\
\hline 15 & 6,5 & 220 & 23 & 3,6 & 200 & 6 & & & & 8 & & \\
\hline 16 & 6,1 & 210 & 25 & & & & & & & 4 & & \\
\hline 17 & 4,9 & 210 & 25 & & & & & & & 16 & & \\
\hline 18 & 4,0 & 220 & 22 & & & & & & & 14 & & \\
\hline 19 & 3,6 & 230 & 22 & & & & & & & 8 & & \\
\hline 20 & 3,4 & 230 & 15 & & & & & & & 6 & & \\
\hline 21 & & & & & & & & & & & & \\
\hline 22 & 3,0 & $25 \overline{5}$ & 11 & & & & & & & 18 & & \\
\hline 23 & & & & & & & & & & & & \\
\hline
\end{tabular}


GENNAIO 1951

\begin{tabular}{|c|c|c|c|c|c|c|c|c|c|c|c|c|}
\hline \multirow{2}{*}{ ORA } & \multicolumn{9}{|c|}{ VALORI MEDIANI MENSILI } & \multicolumn{3}{|c|}{ Percentuale Es } \\
\hline & foF2 & $h^{\prime} F 2$ & val & foF1 & $h^{\prime} F 1$ & val & foE & $h^{\prime} E$ & val & $\cot$ & $>5$ & $>7$ \\
\hline 00 & 3,2 & 270 & 19 & & & & & & & 9 & & \\
\hline 01 & 3,2 & 260 & 22 & & & & & & & & & \\
\hline 02 & 3,2 & 260 & 24 & & & & & & & & & \\
\hline 03 & 3,2 & 250 & 25 & & & & & & & & & \\
\hline 04 & 3,2 & 240 & 26 & & & & & & & & & \\
\hline 05 & 3,2 & 230 & 20 & & & & & & & 4 & & \\
\hline 06 & $(3,0)$ & 230 & 16 & & & & & & & 11 & & \\
\hline 07 & 3,0 & 230 & 19 & & & & & & & 10 & & \\
\hline 08 & 6,0 & 210 & 25 & & & & & & & 4 & & \\
\hline 09 & 6,8 & 210 & 24 & & & & & & & 8 & & \\
\hline 10 & 7,6 & 220 & 23 & 3,5 & 200 & 5 & & & & 4 & & \\
\hline 11 & 8,0 & 220 & 20 & 3,8 & 200 & 9 & & & & 10 & 5 & 5 \\
\hline 12 & 7,6 & 220 & 20 & 3.9 & 200 & 9 & & & & 10 & & \\
\hline 13 & 7.2 & 215 & 21 & 4,0 & 200 & 13 & & & & & & \\
\hline 14 & 7,0 & 220 & 24 & 3,8 & 200 & 13 & & & & & & \\
\hline 15 & 6,6 & 220 & 23 & 3,8 & 200 & 7 & & & & & & \\
\hline 16 & 6,2 & 210 & 23 & & & & & & & 4 & & \\
\hline 17 & 6,0 & 200 & 24 & & & & & & & 4 & & \\
\hline 18 & 4,8 & 220 & 22 & & & & & & & 21 & & \\
\hline 19 & $(4,2)$ & 230 & 19 & & & & & & & 22 & 4 & \\
\hline 20 & 4,0 & 220 & 17 & & & & & & & 24 & & \\
\hline 21 & $(3,5)$ & 230 & 19 & & & & & & & 18 & & \\
\hline 22 & $(3,1)$ & 240 & 22 & & & & & & & 16 & 4 & \\
\hline 23 & 3,0 & 250 & 21 & & & & & & & 26 & & \\
\hline
\end{tabular}

FEBBRAIO 1951

\begin{tabular}{|c|c|c|c|c|c|c|c|c|c|c|c|c|}
\hline \multirow{2}{*}{ ORA } & \multicolumn{9}{|c|}{ VALORI MEDIANI MENSILI } & \multicolumn{3}{|c|}{ Percentuale Es } \\
\hline & foF2 & h'F2 & val & foF1 & h'F1 & val & foE & $h^{\prime} E$ & val & tot & $>5$ & $>7$ \\
\hline 00 & 4,0 & 275 & 15 & & & & & & & & & \\
\hline 01 & 4,0 & 280 & 23 & & & & & & & & & \\
\hline 02 & 3,9 & 275 & 26 & & & & & & & & & \\
\hline 03 & 3,8 & 270 & 26 & & & & & & & & & \\
\hline 04 & 3,8 & 265 & 24 & & & & & & & & & \\
\hline 05 & 3,4 & 250 & 25 & & & & & & & & & \\
\hline 06 & 3,2 & $(250)$ & 16 & & & & & & & & & \\
\hline 07 & 4.3 & 230 & 26 & & & & & & & 4 & & \\
\hline 08 & 6,0 & 210 & 20 & 2,3 & 210 & 5 & & & & & & \\
\hline 09 & 6,5 & 220 & 12 & 3,3 & 205 & 7 & & & & 4 & & \\
\hline 10 & 7,2 & 220 & 12 & 3,3 & 200 & 5 & & & & 4 & & \\
\hline 11 & 8.1 & 230 & 10 & 3,5 & 200 & 0 & & & & & & \\
\hline 12 & 8,1 & 230 & 19 & 3,7 & 200 & 8 & & & & & & \\
\hline 13 & 8,3 & 230 & 20 & 3,6 & 200 & 10 & & & & & & \\
\hline 14 & 8,3 & 230 & 19 & 3,5 & 200 & 8 & & & & 4 & & \\
\hline 15 & 8,0 & 220 & 21 & 3,0 & 200 & 10 & & & & & & \\
\hline 16 & 7,1 & 220 & 18 & 2,9 & 200 & 8 & & & & 4 & & \\
\hline 17 & 5,8 & 210 & 15 & 2,5 & 200 & 6 & & & & 4 & & \\
\hline 18 & $(5,6)$ & (205) & 9 & & & & & & & (6) & & \\
\hline 19 & 5,1 & (220) & 14 & & & & & & & & & \\
\hline 20 & 5,3 & $(230)$ & 10 & & & & & & & 7 & & \\
\hline 21 & $(4,4)$ & (225) & 6 & & & & & & & & & \\
\hline 22 & $(4,2)$ & - & 7 & & & & & & & & & \\
\hline 23 & $(4,2)$ & $(270)$ & 9 & & & & & & & & & \\
\hline
\end{tabular}


MARZO 1951

\begin{tabular}{|c|c|c|c|c|c|c|c|c|c|c|c|c|}
\hline \multirow{2}{*}{ ORA } & \multicolumn{9}{|c|}{ VALORI MEDIANI MENSILI } & \multicolumn{3}{|c|}{ PERCENTUALE ES } \\
\hline & for2 & $h^{\prime} F 2$ & val & foFl & h'F1 & val & foE & $h^{\prime} E$ & $v, 1$ & tot & $>5$ & $>7$ \\
\hline 00 & 4,2 & 280 & 17 & & & & & & & 5 & & \\
\hline 01 & 4.2 & 290 & 17 & & & & & & & & & \\
\hline 02 & 4,1 & 280 & 20 & & & & & & & & & \\
\hline 03 & 4,0 & 280 & 19 & & & & & & & & & \\
\hline 04 & 3,8 & 270 & 17 & & & & & & & & & \\
\hline 05 & 3,6 & 265 & 13 & & & & & & & & & \\
\hline 06 & 4,1 & 240 & 17 & & & & & & & & & \\
\hline 07 & 5,8 & 230 & 23 & & & & & & & & & \\
\hline 08 & 6,4 & 230 & 17 & & & & & & & & & \\
\hline 09 & 7,4 & 245 & 11 & & & & & & & 6 & 6 & \\
\hline 10 & 8,0 & 250 & 13 & & & & & & & 5 & & \\
\hline 11 & 8,4 & 260 & 11 & 3,7 & 200 & 5 & & & & & & \\
\hline 12 & 8,3 & 270 & 18 & 3,8 & $(200)$ & 6, & & & & & & \\
\hline 13 & 8,4 & 260 & 15 & & & & & & & 10) & 5 & \\
\hline 14 & 8,5 & 250 & 17 & 3,8 & $(200)$ & 7 & & & & & & \\
\hline 15 & 8,0 & 240 & 21 & 3,5 & 200 & 8 & & & & & & \\
\hline 16 & 8,0 & 240 & 18 & 3.3 & 210 & 7 & & & & & & \\
\hline 17 & 8,0 & 230 & 22 & & & & & & & 9) & & \\
\hline 18 & 6,3 & 220 & 19 & & & & & & & & & \\
\hline 19 & 6,0 & 220 & 19 & & & & & & & & & \\
\hline 20 & 5,6 & 235 & 17 & & & & & & & & & \\
\hline 21 & 5,2 & 255 & 16 & & & & & & & & & \\
\hline 22 & 5,0 & $(240)$ & 15 & & & & & & & & & \\
\hline 23 & 4,4 & 275 & 13 & & & & & & & & & \\
\hline
\end{tabular}

APRILE 1951

\begin{tabular}{|c|c|c|c|c|c|c|c|c|c|c|c|c|}
\hline \multirow{2}{*}{ ORA } & \multicolumn{9}{|c|}{ VALORI MEDIANI MENSILI } & \multicolumn{3}{|c|}{ Percentlate Es } \\
\hline & foF2 & h'F2 & val & for 1 & h'F1 & val & fo $E$ & $h^{\prime} E$ & val & rot & $2 \cdot 5$ & $>7$ \\
\hline 00 & 5,0 & 300 & 16 & & & & & & & 5 & 5 & \\
\hline 01 & 4.8 & 300 & 16 & & & & & & & 6 & 6 & \\
\hline 02 & 4,5 & 300 & 18 & & & & & & & & & \\
\hline 03 & 4,2 & 280 & 17 & & & & & & & & & \\
\hline 04 & 4,0 & 300 & 17 & & & & & & & & & \\
\hline 05 & 4,3 & 290 & 15 & & & & & & & 5 & & \\
\hline 06 & 5,0 & 250 & 19 & & & & & & & & & \\
\hline 07 & 5,8 & 235 & 18 & 3,0 & 220 & 7 & & & & & & \\
\hline 08 & 6,2 & $(255)$ & 10 & 3,5 & 210 & 6 & & & & & & \\
\hline 09 & $(7,2)$ & $(270)$ & 8 & 3,7 & 200 & 7 & & & & (25) & & \\
\hline 10 & $(7,3)$ & $(255)$ & 8 & 3,8 & 200 & 7 & & & & 9 & & \\
\hline 11 & 8,2 & $(270)$ & 12 & 3,9 & 200 & 7 & & & & 15 & 7 & 7 \\
\hline 12 & 8,0 & 280 & 17 & 4,7 & 200 & 9) & & & & & & \\
\hline 13 & 8.4 & $(270)$ & 15 & 4,5 & 200 & 10 & & & & & & \\
\hline 14 & 8,4 & 270 & 20 & 4.3 & 200 & 12 & & & & 5 & & \\
\hline 15 & 8,4 & 260 & 20 & 3,9 & 200 & 10 & & & & & & \\
\hline 16 & 8,3 & 260 & 20 & 3,5 & 200 & 9 & & & & & & \\
\hline 17 & 8,5 & 250 & 19 & 2.8 & 225 & 7 & & & & 10 & 5 & \\
\hline 18 & 8,4 & 240 & 18 & & & & & & & 11 & 5 & 5 \\
\hline 19 & 7,4 & 220 & 19 & & & & & & & 5 & & \\
\hline 20 & 6,0 & 220 & 16 & & & & & & & & & \\
\hline 21 & 5,3 & $2+5$ & 16 & & & & & & & & & \\
\hline 22 & 5,2 & 280 & 16 & & & & & & & 12 & & \\
\hline 23 & 5,0 & 310 & 11 & & & & & & & & & \\
\hline
\end{tabular}


MAGISIO 1951

\begin{tabular}{|c|c|c|c|c|c|c|c|c|c|c|c|c|}
\hline \multirow{2}{*}{ ORA } & \multicolumn{9}{|c|}{ VALORI MEDIANI MENSILI } & \multicolumn{3}{|c|}{ Percilantunle Es } \\
\hline & for 2 & $h^{\prime} F 2$ & val & $f_{0} F=1$ & $h^{\prime} F 1$ & vill & foE & $h^{\prime} E$ & val & tot & $>5$ & $>7$ \\
\hline 00 & 6,2 & 290 & 22 & & & & & & & 8 & 8 & \\
\hline 01 & 0,0 & 290 & 25 & & & & & & & 11 & 7 & \\
\hline 02 & 5,6 & $30) 1$ & 22 & & & & & & & 12 & \pm & 4 \\
\hline 03 & 5,3 & 2801 & 21 & & & & & & & 8 & 5 & \\
\hline 04 & 5,2 & 270 & 22 & & & & & & & 9 & 5 & 5 \\
\hline 05 & 5,0 & 270) & 24 & & & & & & & 23 & 12 & 4 \\
\hline 06 & 5,6 & 280 & 29 & & & & & & & 28 & 14 & \\
\hline 07 & 6,1 & 275 & 26 & 3,7 & 220) & 12 & & & & 31 & 15 & \\
\hline 08 & 7,0 & 270 & 23 & 4,1 & 200 & 13 & & & & 35 & 21 & 17 \\
\hline 09 & 7,2 & 280 & 14 & 4,4 & 200 & 8 & & & & 50) & 40 & 20) \\
\hline 10) & 7,4 & 280 & 17 & 4,5 & 200 & 9 & & & & 30) & 30 & 10 \\
\hline 11 & 8,0 & 320 & 16 & 5,0 & 200 & 11 & & & & 21 & 21 & 10) \\
\hline 12 & 7,9 & 300 & 20 & 4,9 & 200 & 16 & & & & 41 & 32 & 18 \\
\hline 13 & 8,2 & 300 & 24 & 5,0 & 200 & 19 & & & & 23 & 15 & \\
\hline 14 & 8,5 & 300 & 25 & 4,8 & 200 & 17 & & & & 15 & 15 & 4 \\
\hline 15 & 8,0 & 300 & 24 & 4,6 & 200 & 16 & & & & 36 & 32 & 14 \\
\hline 16 & 7,8 & 280 & 26 & 4,2 & 200 & 11 & & & & 50 & 29 & 21 \\
\hline 17 & 8,1 & 280 & 27 & & & & & & & 46 & 29 & 21 \\
\hline 18 & 8,4 & 2601 & 28 & & & & & & & 50 & 33 & 13 \\
\hline 19 & 8.5 & 2601 & 28 & & & & & & & 36 & 20 & 13 \\
\hline 20 & 8,0 & 245 & 26 & & & & & & & 23 & 12 & 4 \\
\hline 21 & 7,5 & 250 & 22 & & & & & & & 30 & 26 & 9 \\
\hline 22 & 7,0 & 2001 & 26 & & & & & & & 19 & 15 & \\
\hline 23 & 6,8 & 280 & 23 & & & & & & & 15 & 15 & 8 \\
\hline
\end{tabular}

GIUGNO 1951

\begin{tabular}{|c|c|c|c|c|c|c|c|c|c|c|c|c|}
\hline \multirow{2}{*}{ ORA } & \multicolumn{9}{|c|}{$\forall+10 R 1$} & \multicolumn{3}{|c|}{ Percentuale Es } \\
\hline & foF 2 & h'F? & $v, 1$ & for 1 & $h^{\prime} \mathrm{F} 1$ & val & foE & $h^{\prime} E$ & val & tot & $>5$ & $>7$ \\
\hline 00 & 7,0 & 280 & 15 & & & & & & & 6 & 6 & \\
\hline 01 & 6,4 & 290 & 18 & & & & & & & 14 & 9 & 5 \\
\hline 02 & 6,1 & 280 & $1 x$ & & & & & & & 10 & 10 & \\
\hline 03 & 6,4 & 290 & 16 & & & & & & & 10 & 10 & 5 \\
\hline 04 & 5,5 & 265 & 19) & & & & & & & 27 & 18 & 9 \\
\hline 05 & 5,6 & 2611 & 24 & & & & & & & 32 & 12 & 4 \\
\hline 06 & 6,5 & 270 & 23 & 3,5 & 220) & 10 & & & & 54 & 19 & 8 \\
\hline 07 & 7,0 & $251)$ & 23 & 3,8 & 210 & 10 & & & & 54 & +2 & 11 \\
\hline Os & 7,8 & 280 & 21 & 4,2 & 200 & 8 & & & & 54 & +2 & 17 \\
\hline 09 & 7,7 & $27(1)$ & 14 & 4,3 & 200 & 5 & & & & 53 & +2 & 16 \\
\hline 10 & 8,0 & 295 & 15 & & & & & & & 31 & 31 & 12 \\
\hline 11 & 8,5 & 280 & 12 & 5,0 & 200 & 5 & & & & 29 & 29 & 14 \\
\hline 12 & 8,1 & 280 & 17 & & & & & & & 22 & 17 & 6 \\
\hline 13 & 7,8 & 315 & 17 & 5,0 & 200 & 11 & & & & 35 & 35 & 15 \\
\hline 14 & 7,5 & 305 & 19 & 4,8 & 200 & 12 & & & & 35 & 30) & 10 \\
\hline 15 & 8,0 & $30 n$ & 22 & 4.6 & 200 & 12 & & & & 30 & 30) & 13 \\
\hline 16 & 7,9 & 280 & 19 & 4,5 & 200 & 11 & & & & 20 & 20) & 5 \\
\hline 17 & 7,6 & 280 & 20) & 4.4 & 2010 & 9 & & & & 35 & 26 & 9 \\
\hline 18 & 7,8 & 270 & 20) & & & & & & & 57 & 35 & 26 \\
\hline 19 & 8,0 & 2601 & 17 & & & & & & & 40 & 30 & 15 \\
\hline 20 & $(8,0)$ & $(250)$ & 7 & & & & & & & $(+3)$ & (29) & $(14)$ \\
\hline 21 & $(8,0)$ & - & 6 & & & & & & & $(40)$ & $(40)$ & \\
\hline 22 & - & - & - & & & & & & & $\begin{array}{l}(+10) \\
(1+1)\end{array}$ & (t) & $\begin{array}{l}(40) \\
(14)\end{array}$ \\
\hline 23 & - & - & - & & & & & & & $(1+)$ & $(1+)$ & \\
\hline
\end{tabular}


LUGLIO 1951

\begin{tabular}{|c|c|c|c|c|c|c|c|c|c|c|c|c|}
\hline \multirow{2}{*}{ UKA } & \multicolumn{9}{|c|}{ VALORI MEDIANI MENSILI } & \multicolumn{3}{|c|}{ Perchintualf Es } \\
\hline & foF 2 & $h^{\prime} F 2$ & val & foFl & l'ㄷI & h'E & foE & vil & val & tot & $>5$ & $>7$ \\
\hline 00 & $(6,9)$ & $(280)$ & 8 & & & & & & & 67 & 50 & 8 \\
\hline 01 & $(6,8)$ & $(280)$ & 7 & & & & & & & 67 & 50 & 16 \\
\hline 02 & $(6,5)$ & $280^{\circ}$ & 11 & & & & & & & +4 & 31 & 12 \\
\hline 03 & $(6,9)$ & (280) & 8 & & & & & & & +6 & 38 & 8 \\
\hline 04 & $6,6^{\circ}$ & 280 & 10 & & & & & & & 62 & 31 & 8 \\
\hline 05 & $(6,0)$ & $(25 \overline{5})$ & 9 & & & & & & & 93 & 47 & 7 \\
\hline 06 & 5,9 & 270 & 20 & 3,8 & 230 & 12 & 2,8 & 100 & 5 & +2 & 21 & 8 \\
\hline 07 & 6,9 & 270 & 20 & 4,2 & 220 & 15 & 2,8 & 105 & 7 & 88 & 37 & 17 \\
\hline 08 & 7.2 & 280 & 16 & 4,8 & 210 & 11 & 3,2 & 100 & 5 & 80 & 40 & 10 \\
\hline 09 & 7.1 & 305 & 18 & 5,0 & 200 & 12 & & & & 80 & 80 & 15 \\
\hline 10 & 6,8 & 310 & 18 & 5,0 & 205 & 14 & & & & 70 & 60 & 15 \\
\hline 11 & 7,5 & 310 & 15 & 5,0 & 200 & 10 & & & & 83 & 72 & 28 \\
\hline 12 & 7,2 & 330 & 17 & 5,0 & 220 & 13 & & & & 65 & 55 & 40 \\
\hline 13 & 7.2 & 320 & 20 & 5,0 & 220 & 16 & & & & 72 & 62 & 28 \\
\hline 14 & 7,0 & 320 & 20 & 4,9 & 205 & 19 & & & & 67 & 57 & 14 \\
\hline 15 & 7,0 & 310 & 20) & 4,8 & 220 & 17 & 3,3 & 100 & 5 & 52 & 48 & 22 \\
\hline 16 & 7,2 & 300 & 21 & 4.7 & 220 & 19 & 3,3 & 100 & 5 & 68 & 36 & 18 \\
\hline 17 & 7,1 & 290 & 22 & 4,5 & 225 & 18 & & & & 65 & 35 & 4 \\
\hline 18 & 7,0 & 280 & 21 & 4,0 & 225 & 12 & & & & 64 & 41 & \\
\hline 19 & 7,1 & 260 & 19 & & & & & & & 67 & 38 & 14 \\
\hline 20 & 7,7 & 250 & 10 & & & & & & & 87 & 60 & 20 \\
\hline 21 & $(7,7)$ & 260 & 12 & & & & & & & 71 & 53 & 6 \\
\hline 22 & - & - & - & & & & & & & 100 & 80 & \\
\hline 23 & $(7,0)$ & $(260)$ & 6 & & & & & & & 80 & 67 & 7 \\
\hline
\end{tabular}

AGOSTO 1951

\begin{tabular}{|c|c|c|c|c|c|c|c|c|c|c|c|c|}
\hline \multirow{2}{*}{ ORA } & \multicolumn{9}{|c|}{ VALORI MEDIANI MENSILI } & \multicolumn{3}{|c|}{ P'ercentuatre Es } \\
\hline & for2 & h'F2 & val & foF1 & $\mathrm{l}^{\prime} \mathrm{F} 1$ & vil & foE & $h^{\prime} E$ & val & tot & $>5$ & $>7$ \\
\hline 00 & $(5,4)$ & (320) & 9 & & & & & & & 67 & $4+$ & 17 \\
\hline 01 & $(5,7)$ & 300 & 10 & & & & & & & 60 & 45 & 15 \\
\hline 02 & $(4,9)$ & 290 & 6 & & & & & & & 69 & 56 & 25 \\
\hline 03 & - & - & - & & & & & & & 67 & 58 & 29 \\
\hline 04 & - & (270) & 5 & & & & & & & 75 & 67 & 58 \\
\hline 05 & $(4,4)$ & $(280)$ & 9 & & & & & & & 75 & 38 & \\
\hline 06 & 5,3 & 250 & 21 & & & & & & & 56 & 41 & 7 \\
\hline 07 & 5,8 & 250 & 23 & 4,0 & 220 & 9 & & & & 70 & 43 & 23 \\
\hline 08 & 6,3 & 265 & 21 & 4,3 & 200 & 17 & & & & 83 & 54 & 8 \\
\hline 09 & 6,5 & 280 & 19 & 4,9 & 200 & 15 & & & & 75 & 62 & 17 \\
\hline 10 & 6,5 & 275 & 25 & 4,9 & 200 & 17 & & & & 76 & 62 & 28 \\
\hline 11 & 6,5 & 285 & 22 & 5,0 & 190 & 18 & & & & 95 & 71 & 14 \\
\hline 12 & 6,7 & 300 & 21 & 4,9 & 200 & 18 & & & & 61 & 54 & 21 \\
\hline 13 & 6,8 & 300 & 27 & 4,8 & 200 & 21 & & & & 62 & 45 & 28 \\
\hline 14 & 6,8 & 300 & 28 & 4,7 & 200 & 28 & & & & 38 & 28 & 10 \\
\hline 15 & 6,7 & 280 & 27 & 4,7 & 200 & 27 & & & & 56 & 36 & 4 \\
\hline 16 & 7,0 & 280 & 28 & 4,5 & 200 & 22 & & & & 52 & 48 & 7 \\
\hline 17 & 6,7 & 270 & 26 & 4,3 & 220 & 16 & & - & & 66 & 48 & 14 \\
\hline 18 & 7,1 & 260 & 22 & & & & & & & 63 & 47 & 17 \\
\hline 19 & 7,4 & 260 & 23 & & & & & & & 60 & 43 & 30 \\
\hline 20 & $(8,0)$ & $(260)$ & 9 & & & & & & & 76 & 62 & 38 \\
\hline 21 & $(7,8)$ & 260 & 11 & & & & & & & 61 & 61 & 22 \\
\hline 22 & $(7,1)$ & $(270)$ & 8 & & & & & & & 65 & 53 & 18 \\
\hline 23 & - & - & - & & & & & & & 67 & 56 & 22 \\
\hline
\end{tabular}


SETTEMBRE 1951

\begin{tabular}{|c|c|c|c|c|c|c|c|c|c|c|c|c|}
\hline \multirow{2}{*}{ ORA } & \multicolumn{9}{|c|}{ VALORI MEDIANI MENSILI } & \multicolumn{3}{|c|}{ |'ercentuale Es } \\
\hline & for 2 & h'F2 & val & for1 & $h^{\prime} \mathrm{F} 1$ & val & foE & $h^{\prime} E$ & val & $\operatorname{tot}$ & $>5$ & $>7$ \\
\hline 00 & $(5,0)$ & $(280)$ & 9 & & & & & & & & & \\
\hline 01 & 5,2 & 300 & 11 & & & & & & & 9 & & \\
\hline 02 & $(4,6)$ & 280 & 11 & & & & & & & 8 & & \\
\hline 03 & 4,8 & 280 & 10 & & & & & & & 10 & & \\
\hline 04 & $(3,9)$ & (300) & 8 & & & & & & & (11) & & \\
\hline 05 & $(3,5)$ & 280 & 10) & & & & & & & $25^{\prime}$ & & \\
\hline 06 & $(5,2)$ & $(2+5)$ & 8 & & & & & & & (22) & & \\
\hline 07 & 6,7 & 250 & 17 & 3,7 & 210 & 5 & & & & 17 & 11 & 6 \\
\hline 08 & 6,5 & 245 & 16 & 3,8 & 200 & 9 & & & & 39 & 17 & \\
\hline 09 & 7,4 & 255 & 18 & 4,2 & 200 & 17 & & & & 20 & 10 & 5 \\
\hline 10 & 7,2 & 255 & 16 & 4,4 & 200 & 16 & & & & 26 & 16 & 5 \\
\hline 11 & 7,6 & 250 & 15 & 4,9 & 200 & 15 & & & & +2 & 26 & 5 \\
\hline 12 & 7,7 & 270) & 18 & 4,9 & 200 & 17 & & & & 55 & 30 & 20 \\
\hline 13 & 7,8 & 270 & 19 & 5,0 & 200 & 20 & & & & 22 & 18 & \\
\hline 14 & 7,4 & 260 & 20 & 4,8 & 200 & 18 & & & & 27 & 22 & 5 \\
\hline 15 & 7,4 & 260 & 22 & 4,5 & 200 & 22 & & & & 18 & 5 & \\
\hline 16 & 7,5 & 260 & 19 & 4,1 & 210 & 13 & & & & 21 & 11 & \\
\hline 17 & 7,5 & 260 & 23 & 3,7 & 215 & 6 & & & & 13 & 4 & \\
\hline 18 & 7,5 & 245 & 20 & & & & & & & 19 & 5 & 5 \\
\hline 19 & 8.0 & 250 & 17 & & & & & & & 26 & 5 & \\
\hline 20 & 6,9 & 250 & 14 & & & & & & & 13 & 13 & \\
\hline 21 & 6.1 & 250 & 11 & & & & & & & 23 & 15 & 8 \\
\hline 22 & 0,0 & 250 & 11 & & & & & & & 40 & 7 & 7 \\
\hline 23 & $(4,8)$ & $(280)$ & 9 & & & & & & & 27 & 9 & \\
\hline
\end{tabular}

OTTOBRE 1951

\begin{tabular}{|c|c|c|c|c|c|c|c|c|c|c|c|c|}
\hline \multirow{2}{*}{ ORA } & \multicolumn{9}{|c|}{ VALORI MEDIANI MENSILI } & \multicolumn{3}{|c|}{ Percentuale Es } \\
\hline & foF2 & h'F2 & val & foF1 & h'F1 & val & foE & h'E & val & tot & $>5$ & $>7$ \\
\hline (1) & 4,0 & 295 & 23 & & & & & & & 17 & 4 & \\
\hline 01 & 3,8 & 300 & 24 & & & & & & & 20 & & \\
\hline 02 & 3,8 & 300 & 24 & & & & & & & 15 & 4 & \\
\hline 03 & 3,8 & 290 & 27 & & & & & & & 28 & 7 & \\
\hline 04 & 3,8 & 270 & 27 & & & & & & & & & \\
\hline 05 & 3,7 & 250 & 26 & & & & & & & & & \\
\hline 06 & 3,9 & 250 & 16 & & & & & & & & & \\
\hline 07 & 5,3 & 230 & 25 & & & & & & & & & \\
\hline 08 & 7,0 & 230 & 26 & 3,6 & 210 & 14 & & & & 11 & & \\
\hline (19) & 7,9 & 240 & 24 & 3,6 & 200 & 19 & & & & 12 & 4 & \\
\hline 10 & 8,2 & 240 & 23 & 3,9 & 200 & 11 & & & & 35 & 22 & \\
\hline 11 & 8.2 & 240 & 25 & 4,0 & 200 & 12 & & & & 35 & 19 & \\
\hline 12 & 8,2 & 250 & 23 & 4,0 & 200 & 18 & & & & 29 & 12 & \\
\hline 13 & 8,2 & 240 & 26 & 3,8 & 200 & 16 & & & & 35 & 15 & 4 \\
\hline 14 & 8,5 & 240 & 25 & 3,8 & 200 & 15 & & & & 16 & 8 & 4 \\
\hline 15 & 8,5 & $2+0$ & 25 & 3,6 & 220 & 15 & & & & 16 & 4 & \\
\hline 16 & 8,5 & 240 & 26 & 3,5 & 220 & 13 & & & & 23 & 4 & \\
\hline 17 & 8,1 & 230 & 25 & & & & & & & 28 & 4 & \\
\hline 18 & 6,4 & 220 & 23 & & & & & & & 22 & 9 & \\
\hline 19 & 5,5 & 240 & 21 & & & & & & & 22 & 19 & 9 \\
\hline 2 & 5,2 & 250 & 18 & & & & & & & 35 & 10 & 5 \\
\hline 21 & 4,6 & 270 & 13 & & & & & & & 26 & 11 & 5 \\
\hline 22 & $(4,3)$ & 280 & 15 & & & & & & & 26 & 21 & 5 \\
\hline 23 & $(4,2)$ & 280 & 14 & & & & & & & 12 & 6 & \\
\hline
\end{tabular}


NOVEMBRE 1951

\begin{tabular}{|c|c|c|c|c|c|c|c|c|c|c|c|c|}
\hline \multirow{2}{*}{ ORA } & \multicolumn{9}{|c|}{ VA1ORE MEDIANI MENSELE } & \multicolumn{3}{|c|}{ P'ercentuale Ev } \\
\hline & BOF2 & $h^{\prime} F 2$ & val & foFl & h'FI & val & foE & h'E & val & tot & $>5$ & $>7$ \\
\hline 00 & $(3,5)$ & - & 5 & & & & & & & $(16)$ & & \\
\hline 01 & 3,5 & 3111 & 11 & & & & & & & & & \\
\hline 02 & 3.8 & 2711 & 10) & & & & & & & & & \\
\hline 03 & 3,8 & 270 & 12 & & & & & & & 9 & & \\
\hline $0+$ &,+ 0 & 250 & 12 & & & & & & & & & \\
\hline 05 & $(3,7)$ & $(250)$ & 9 & & & & & & & 111 & & \\
\hline 06 & - & - & - & & & & & & & & & \\
\hline 07 & 5,2 & $2+1)$ & 23 & & & & & & & $\begin{array}{r}9 \\
+\end{array}$ & 4 & 4 \\
\hline 08 & 7,0 & 225 & 26 & 3.6 & $\begin{array}{l}2(111) \\
2011\end{array}$ & 5 & & & & & & \\
\hline 09 & 7,7 & 230 & 27 & 3.3 & $\begin{array}{l}2 \\
20010\end{array}$ & 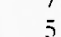 & & & & 10 & 7 & 4 \\
\hline 10 & 8,1 & 2311 & $2 \pi$ & 4.11 & $\begin{array}{l}2010 \\
20010\end{array}$ & (i) & & & & & & \\
\hline 11 & 8,2 & 230) & 28 & 4.1 & & 15 & & & & 111 & & \\
\hline 12 & 8,5 & 230 & $2 \pi$ &,+ 3 & 2001 & 12 & & & & + & & \\
\hline 13 & 8,2 & 230 & 28 & +.2 & 2001 & $\overrightarrow{9}$ & & & & & & \\
\hline 14 & $\begin{array}{l}8,0 \\
880\end{array}$ & $\begin{array}{l}230 \\
230\end{array}$ & 29) & 4.2 & 200 & 5 & & & & 7 & 4 & \\
\hline $\begin{array}{l}15 \\
16\end{array}$ & $\begin{array}{l}8,0 \\
7,6\end{array}$ & $\begin{array}{l}230 \\
230\end{array}$ & $\begin{array}{l}29 \\
2 x\end{array}$ & & & & & & & 7 & 4 & \\
\hline 17 & 5,8 & 220 & 20 & & & & & & & 15 & 10 & 5 \\
\hline 18 & 5,0 & 230 & 16 & & & & & & & 19 & 6 & \\
\hline 19 & 4,9 & 240 & 16 & & & & & & & 6 & 6 & \\
\hline 20 & $(4,3)$ & $(250)$ & 8 & & & & & & & (22) & (11) & \\
\hline 21 & - & - & - & & & & & & & & & \\
\hline 22 & - & - & - & & & & & & & & & \\
\hline 23 & - & - & - & & & & & & & & & \\
\hline
\end{tabular}

DICEMBRE 1951

\begin{tabular}{|c|c|c|c|c|c|c|c|c|c|c|c|c|}
\hline \multirow{2}{*}{ ORA } & \multicolumn{9}{|c|}{ VALORI MEDIANI MENSILL } & \multicolumn{3}{|c|}{ Percentchale E; } \\
\hline & $f \circ F 2$ & $h^{\prime} F 2$ & ral & foF1 & $h^{\prime} F 1$ & val & $f(0)$ & $h^{\prime} E$ & val & $\operatorname{tot}$ & $>5$ & 7.7 \\
\hline 00 & $(3,7)$ & - & 5 & & & & & & & & & \\
\hline 01 & $(3,4)$ & $(275)$ & 8 & & & & & & & & & \\
\hline 02 & $(3,7)$ & (280) & 9) & & & & & & & & & \\
\hline 03 & 3,5 & 270 & 11 & & & & & & & & & \\
\hline 04 & $(3, \overline{3})$ & $(250)$ & 9 & & & & & & & & & \\
\hline 05 & - & - & - & & & & & & & & & \\
\hline 06 & - & - & - & & & & & & & & 9 & \\
\hline 07 & 3,5 & $241)$ & 10) & & & & & & & 5 & & \\
\hline 08 & 6,0 & 220 & 19 & 3,3 & 2(1) & 8 & & & & $\frac{3}{5}$ & & \\
\hline 09 & 6,9 & 220 & 20 & 3.5 & 200 & 11 & & & & $\frac{5}{5}$ & & \\
\hline 10 & 8,0 & 220 & 19 & 3,3 & 200 & 11 & & & & 5 & 3 & \\
\hline 11 & 8,5 & 225 & 20 & 4,0 & 200 & 9 & & & & 5 & & \\
\hline 12 & 7,7 & 230) & 21 & 4,0 & 2010 & 13 & & & & 5 & 5 & \\
\hline 13 & 8,0 & 230 & 20 & 4,0 & 200 & 11 & & & & i) & 3 & \\
\hline 14 & 7,6 & 230) & 22 & & & & & & & 5 & & \\
\hline 15 & 7.8 & 220 & 20) & & & & & & & 9 & & \\
\hline 16 & 6,8 & 210 & 22 & 3,4 & 200 & 6 & & & & 14 & & \\
\hline 17 & 5,5 & 220 & 20 & & & & & & & 7 & & \\
\hline 18 & 5,2 & 230 & 13 & & & & & & & (22) & & \\
\hline 19 & $(4,5)$ & $(250)$ & 7 & & & & & & & (16) & (16) & \\
\hline $\begin{array}{l}20 \\
21\end{array}$ & - & $-(250)$ & $\underline{3}$ & & & & & & & & & \\
\hline 32 & - & - & - & & & & & & & & & \\
\hline 23 & - & - & - & & & & & & & & & \\
\hline
\end{tabular}




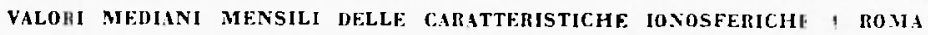

GENNAIO 1952

\begin{tabular}{|c|c|c|c|c|c|c|c|c|c|c|c|c|}
\hline \multirow{2}{*}{ ORA } & \multicolumn{9}{|c|}{ VALORI MEDIANI MENSILI } & \multicolumn{3}{|c|}{ Percentuale Es } \\
\hline & foF2 & $h^{\prime} F 2$ & val & forl 1 & $h^{\prime} F 1$ & val & foE & $h^{\prime} E$ & val & tot & $>5$ & $>7$ \\
\hline 00 & $(3,5)$ & $(265)$ & 8 & & & & & & & & & \\
\hline 01 & 3,5 & 270 & 11 & & & & & & & & & \\
\hline 02 & 3,5 & 265 & 12 & & & & & & & & & \\
\hline 03 & 3,5 & 260 & 13 & & & & & & & & & \\
\hline 04 & 3,4 & 250 & 12 & & & & & & & & & \\
\hline 05 & 3,1 & 245 & 12 & & & & & & & & & \\
\hline 06 & $(2,9)$ & (230) & 9 & & & & & & & & & \\
\hline 07 & 3,5 & 220 & 10 & & & & & & & & & \\
\hline 08 & 5,5 & 220 & 28 & 3,1 & 200 & 15 & & & & & & \\
\hline 09 & 6,7 & 210 & 26 & 3,5 & 200 & 15 & & & & & & \\
\hline 10 & 8,3 & 220 & 27 & 3,8 & 200 & 13 & & & & & & \\
\hline 11 & 8,0 & 230 & 24 & 4,0 & 200 & 9 & & & & & & \\
\hline 12 & 7,4 & 220 & 24 & 4,0 & 200 & 16 & & & & & & \\
\hline 13 & 7,2 & 230 & 28 & 3,9 & 200 & 20 & & & & 7 & & \\
\hline 14 & 7,1 & 230 & 26 & 3,6 & 200 & 15 & & & & & & \\
\hline 15 & 7,5 & 220 & 29 & 3,0 & 200 & 16 & & & & & & \\
\hline 16 & 6,4 & 220 & 29 & 3.3 & 200 & 14 & & & & & & \\
\hline 17 & 5,5 & 210 & 29 & & & & & & & 3 & 3 & \\
\hline 18 & 5,2 & 220 & 20 & & & & & & & 5 & & \\
\hline 19 & 4,9 & 230 & 14 & & & & & & & 14 & & \\
\hline 20 & $(3,8)$ & (230) & 7 & & & & & & & 7 & & \\
\hline 21 & - & - & - & & & & & & & (20) & & \\
\hline 22 & - & $(270)$ & 6 & & & & & & & (14) & & \\
\hline 23 & $(3,4)$ & (270) & 6 & & & & & & & (14) & & \\
\hline
\end{tabular}

FEBBRAIO 1952

\begin{tabular}{|c|c|c|c|c|c|c|c|c|c|c|c|c|}
\hline \multirow{2}{*}{ ORA } & \multicolumn{9}{|c|}{ VALORI MEDIANI MENSILI } & \multicolumn{3}{|c|}{ Percentuale Es } \\
\hline & foF2 & $h^{\prime} F 2$ & val & foFl & h'F1 & val & foE & $h^{\prime} E$ & val & tot & $>5$ & $>7$ \\
\hline 00 & 3,5 & 280 & 17 & & & & & & & 6 & & \\
\hline 01 & 3,5 & 280 & 16 & & & & & & & 11 & & \\
\hline 02 & 3,5 & 280 & 17 & & & & & & & 6 & & \\
\hline 03 & 3,4 & 270 & 17 & & & & & & & & & \\
\hline 04 & 3,4 & 280 & 15 & & & & & & & & & \\
\hline 05 & 3,0 & 260 & 16 & & & & & & & & & \\
\hline 06 & 2,9 & 250 & 14 & & & & & & & & & \\
\hline 07 & 3,8 & 230 & 17 & & & & & & & & & \\
\hline 08 & 5,7 & 220 & 17 & & & & & & & & & \\
\hline 09 & 6,7 & 220 & 19 & & & & & & & & & \\
\hline 10 & 7,1 & 220 & 19 & 4,0 & 200 & & & & & 5 & & \\
\hline 11 & 7,2 & 230 & 18 & 4,2 & 200 & & & & & 5 & & \\
\hline 12 & 7,8 & 230 & 16 & 4,3 & 200 & & & & & 6 & & 6 \\
\hline 13 & 7,1 & 230 & 19 & 4,3 & 200 & & & & & 5 & & \\
\hline 14 & 6,5 & 230 & 19 & 4,2 & 205 & 12 & & & & 5 & & \\
\hline 15 & 7,0 & 230 & 18 & 3,8 & 205 & & & & & 5 & & \\
\hline 16 & 6,5 & 220 & 17 & 3,6 & 205 & & & & & & & \\
\hline 17 & 6,1 & 220 & 17 & & & & & & & 6 & & \\
\hline 18 & 5,5 & 220 & 15 & & & & & & & 6 & & 6 \\
\hline 19 & 5,1 & 230 & 16 & & & & & & & 30 & & \\
\hline 20 & 4,3 & 240 & 17 & & & & & & & 12 & & \\
\hline 21 & 4,0 & 260 & 13 & & & & & & & 19 & & \\
\hline 22 & 3,8 & 270 & 13 & & & & & & & 13 & & \\
\hline 23 & 3,5 & 280 & 16 & & & & & & & 6 & & \\
\hline
\end{tabular}


NOVEMBRE 1952

\begin{tabular}{|c|c|c|c|c|c|c|c|c|c|c|c|c|}
\hline \multirow{2}{*}{ ORA } & \multicolumn{9}{|c|}{ VALORI MEDIANI MENSILI } & \multicolumn{3}{|c|}{ Percentuale Es } \\
\hline & foF 2 & h'F2 & val & foF1 & h'F1 & val & foE & $h^{\prime} E$ & val & tot & $>5$ & $>7$ \\
\hline 00 & 3,1 & 240 & 22 & & & & & & & 4 & & \\
\hline 01 & 3,2 & 250 & 23 & & & & & & & & & \\
\hline 02 & 3,2 & 250 & 20 & & & & & & & 5 & & \\
\hline 03 & 3,2 & 240 & 21 & & & & & & & 5 & & \\
\hline 04 & 3,3 & 235 & 20 & & & & & & & & & \\
\hline 05 & 3,3 & 210 & 19 & & & & & & & & & \\
\hline 06 & 3,2 & 210 & 18 & & & & & & & 5 & & \\
\hline 07 & 4,0 & 205 & 23 & & & & & & & 4 & & \\
\hline 08 & 6,0 & 200 & 22 & & & & & & & & & \\
\hline 09 & 6,3 & 200 & 22 & 3,8 & 200 & 9 & 2,7 & 100 & 6 & 5 & & \\
\hline 10 & 6,9 & 220 & 21 & 4,0 & 200 & 9 & 3,0 & 100 & 9 & 5 & & \\
\hline 11 & 7,0 & 220 & 21 & 3,8 & 200 & 15 & 3,2 & 100 & 11 & 9 & & \\
\hline 12 & 7,0 & 210 & 22 & 4,0 & 200 & 10 & 3,3 & 100 & 5 & 5 & 5 & \\
\hline 13 & 6,8 & 210 & 22 & 3,9 & 200 & 6 & 3,2 & 100 & 7 & 5 & & \\
\hline 14 & 6,5 & 205 & 23 & 3,9 & 200 & 6 & 3,1 & 100 & 5 & 8 & & \\
\hline 15 & 6,0 & 210 & 24 & & & & & & & & & \\
\hline 16 & 6,0 & 200 & 24 & & & & 2,4 & 100 & 7 & & & \\
\hline 17 & 5,0 & 200 & 22 & & & & & & & 5 & & \\
\hline 18 & 3,9 & 215 & 18 & & & & & & & 5 & 5 & \\
\hline 19 & 3,8 & 215 & 17 & & & & & & & 6 & 6 & \\
\hline 20 & 3,4 & 230 & 19 & & & & & & & 24 & 10 & \\
\hline 21 & 3,3 & 230 & 21 & & & & & & & 24 & 5 & \\
\hline 22 & 3,2 & 240 & 16 & & & & & & & 6 & & \\
\hline 23 & 3,2 & 240 & 17 & & & & & & & & & \\
\hline
\end{tabular}

DICEMBRE 1952

\begin{tabular}{|c|c|c|c|c|c|c|c|c|c|c|c|c|}
\hline \multirow{2}{*}{ ORA } & \multicolumn{9}{|c|}{ VALORI MEDIANI MENSILI } & \multicolumn{3}{|c|}{ Percentuale Es } \\
\hline & foF2 & h'F2 & val & foF1 & h'F1 & val & foE & $h^{\prime} E$ & val & tot & $>5$ & $>7$ \\
\hline 00 & 3,3 & 240 & 26 & & & & & & & & & \\
\hline 01 & 3,3 & 230 & 26 & & & & & & & & & \\
\hline 02 & 3,3 & 240 & 29 & & & & & & & 3 & & \\
\hline 03 & 3,2 & 240 & 28 & & & & & & & 3 & & \\
\hline 04 & 3,2 & 230 & 29 & & & & & & & & & \\
\hline 05 & 3,0 & 215 & 28 & & & & & & & 3 & & \\
\hline 06 & 3,4 & 210 & 23 & & & & & & & & & \\
\hline 07 & 3,5 & 205 & 27 & 3,0 & 200 & 5 & & & & 14 & & \\
\hline 08 & 5,2 & 200 & 29 & 3,3 & 190 & 27 & 1,8 & 100 & 26 & 14 & & \\
\hline 09 & 5,8 & 210 & 29 & 3,5 & 190 & 27 & 2,2 & 100 & 25 & 7 & & \\
\hline 10 & 6,5 & 210 & 28 & 3,7 & 190 & 26 & 2,3 & 105 & 23 & 3 & & \\
\hline 11 & 7,0 & 210 & 27 & 3,7 & 190 & 26 & 2,5 & 100 & 21 & 3 & & \\
\hline 12 & 6,7 & 210 & 27 & 3,8 & 200 & 26 & 2,3 & 100 & 25 & 3 & 3 & \\
\hline 13 & 6,2 & 210 & 27 & 3,5 & 190 & 26 & 2,3 & 100 & 25 & 3 & 3 & \\
\hline 14 & 6,0 & 210 & 30 & 3,5 & 200 & 30 & 2,4 & 100 & 29 & 7 & & \\
\hline 15 & 6,4 & 220 & 30 & 3,5 & 200 & 29 & 2,3 & 100 & 29 & & & \\
\hline 16 & 6,0 & 205 & 29 & 3,4 & 190 & 27 & 2,0 & 100 & 27 & 3 & & \\
\hline 17 & 4,6 & 200 & 28 & 3,1 & 190 & 5 & 1,9 & 100 & 7 & & & \\
\hline 18 & 4,1 & 210 & 25 & & & & & & & & & \\
\hline 19 & 3,9 & 220 & 23 & & & & & & & 4 & & \\
\hline 20 & 3,3 & 220 & 25 & & & & & & & & & \\
\hline 21 & 3,3 & 230 & 26 & & & & & & & & & \\
\hline 22 & 3,1 & 230 & 23 & & & & & & & & & \\
\hline 23 & 3,3 & 230 & 24 & & & & & & & & & \\
\hline
\end{tabular}


GENNAIO 1953

\begin{tabular}{|c|c|c|c|c|c|c|c|c|c|c|c|c|}
\hline \multirow{2}{*}{ WN } & \multicolumn{9}{|c|}{ VALORI MEDIANI MENSILI } & \multicolumn{3}{|c|}{ Percentuale Es } \\
\hline & toF2 & $h^{\prime} F 2$ & val & foFl & $h^{\prime} F 1$ & val & foE & $h^{\prime} E$ & val & tot & $>5$ & $>7$ \\
\hline 00 & 3,3 & 240 & 21 & & & & & & & & & \\
\hline 01 & 3,0 & 220 & 22 & & & & & & & & & \\
\hline 02 & 3,0 & 230 & 21 & & & & & & & 9 & & \\
\hline 03 & 3,0 & 230 & 21 & & & & & & & 5 & & \\
\hline 04 & 3,0 & 220 & 23 & & & & & & & 4 & & \\
\hline 05 & 2,9 & 220 & 23 & & & & & & & 4 & & \\
\hline 06 & 2,9 & 220 & 23 & & & & & & & 8 & 4 & \\
\hline 07 & 2,6 & 210 & 23 & & & & & & & 8 & & \\
\hline 08 & 5,0 & 200 & 22 & 3,0 & 200 & 6 & 1,9 & 100 & 7 & 5 & & \\
\hline 09 & 6,1 & 210 & 25 & 3,4 & 200 & 6 & 2,3 & 100 & 5 & 8 & 4 & \\
\hline 10 & 6,8 & 220 & 24 & 3,8 & 200 & 11 & 2,5 & 100 & 9 & 4 & & \\
\hline 11 & 7,0 & 210 & 23 & 3,9 & 195 & 11 & 2,8 & 100 & 9 & 13 & 8 & \\
\hline 12 & 6,7 & 210 & 23 & 4,0 & 190 & 12 & 2,9 & 100 & 12 & 8 & 4 & 4 \\
\hline 13 & 6,2 & 210 & 22 & 4,0 & 195 & 12 & 2,7 & 100 & 10 & & & \\
\hline 14 & 6,0 & 220 & 23 & 3,6 & 190 & 11 & 2.6 & 100 & 10 & 17 & 8 & \\
\hline 15 & 6,0 & 210 & 23 & 3,5 & 195 & 6 & 2,4 & 100 & 8 & 4 & & \\
\hline 16 & 5,8 & 200 & 25 & & & & 2.2 & 100 & 8 & 8 & & \\
\hline 17 & 5,0 & 200 & 25 & & & & & & & 4 & 4 & \\
\hline 18 & 3,9 & 200 & 23 & & & & & & & 8 & & \\
\hline 19 & 3,7 & 210 & 19 & & & & & & & 16 & 5 & \\
\hline 20 & 3,2 & 220 & 17 & & & & & & & 11 & 11 & \\
\hline 21 & 3,1 & 240 & 17 & & & & & & & 6 & & \\
\hline 22 & 3,2 & 250 & 19 & & & & & & & & & \\
\hline 23 & 3,1 & 240 & 22 & & & & & & & & & \\
\hline
\end{tabular}

FEBBRAIO 1953

\begin{tabular}{|c|c|c|c|c|c|c|c|c|c|c|c|c|}
\hline \multirow{2}{*}{ ()RA } & \multicolumn{9}{|c|}{ VALORI MEDIANI MENSILI } & \multicolumn{3}{|c|}{ Percentuale Es } \\
\hline & for2 & $h^{\prime} F 2$ & vil & foF1 & $h^{\prime} F 1$ & val & fo $E$ & $h^{\prime} E$ & val & tot & $>5$ & $>7$ \\
\hline 00 & 3,0 & 250 & 26 & & & & & & & & & \\
\hline 01 & 3,1 & 250 & 25 & & & & & & & & & \\
\hline 02 & 3,0 & 250 & 25 & & & & & & & & & \\
\hline 03 & 3,0 & 250 & 25 & & & & & & & & & \\
\hline 04 & 3,0 & 250 & 25 & & & & & & & & & \\
\hline 05 & 3,0 & 230 & 25 & & & & & & & & & \\
\hline 06 & 2,7 & 230 & 25 & & & & & & & & & \\
\hline 07 & 3,3 & 210 & 25 & & & & & & & & & \\
\hline 08 & 5,2 & 200 & 25 & 3,0 & 200 & 8 & 2.0 & 110 & 7 & & & \\
\hline 09 & 5.4 & 210 & 24 & 3,4 & 190 & 16 & 2.4 & 105 & 7 & 4 & & \\
\hline 10 & 5,8 & 220 & 23 & 3,7 & 190 & 20) & 2,8 & 100 & 10 & 4 & 4 & 4 \\
\hline 11 & 6,0 & 230 & 23 & 3,9 & 190 & 22 & 3.0 & 100 & 14 & 8 & 4 & \\
\hline 12 & 6,3 & 235 & 24 & 3,8 & 190 & 24 & 3,1 & 100 & 20 & 4 & & \\
\hline 13 & 6.1 & 230) & 23 & 4.0 & 200 & 2.3 & 3,0 & 100 & 22 & & & \\
\hline 14 & 5,8 & 230 & 25 & 3,8 & 190 & 24 & 2,8 & 100 & 21 & & & \\
\hline 15 & 5,7 & 230 & 25 & 3,7 & 190 & 23 & 2.7 & 100 & 18 & & & \\
\hline 16 & 5,5 & 220 & 26 & 3,3 & $2(x)$ & 12 & 2,5 & 105 & 14 & & & \\
\hline 17 & 5,3 & 200 & 27 & & & & 2.2 & 105 & 11 & 7 & & \\
\hline 18 & 4,4 & 200 & 27 & & & & & & & 7 & 4 & \\
\hline 19 & 3,5 & 225 & 27 & & & & & & & 14 & & \\
\hline 20 & 3.5 & 220 & 27 & & & & & & & 14 & & \\
\hline 21 & 3,3 & 210 & 27 & & & & & & & 14 & & \\
\hline 22 & 3,0 & 230 & 27 & & & & & & & 18 & & \\
\hline 23 & 3,0 & 230 & 27 & & & & & & & 7 & & \\
\hline
\end{tabular}


MARZO 1953

\begin{tabular}{|c|c|c|c|c|c|c|c|c|c|c|c|c|}
\hline \multirow{2}{*}{ ORA } & \multicolumn{9}{|c|}{ VALORI MEDIANI MENSILI } & \multicolumn{3}{|c|}{ Plircentuale Es } \\
\hline & foF2 & h'F2 & val & fort & h'Fi & val & fiv $E$ & h'E & val & tot & $>5$ & $>7$ \\
\hline 00 & 3,2 & 250 & 25 & & & & & & & 12 & + & \\
\hline 01 & 3,1 & 260 & 26 & & & & & & & 12 & & \\
\hline 02 & 3,0 & 260 & 26 & & & & & & & 12 & & \\
\hline 03 & 2,9 & 260 & 25 & & & & & & & + & & \\
\hline 04 & 2,8 & 250 & 25 & & & & & & & & & \\
\hline 05 & 2,7 & 240 & 25 & & & & & & & 8 & 4 & \\
\hline 06 & 2,7 & 225 & 25 & & & & & & & 8 & & \\
\hline 07 & 4,1 & 205 & 26 & & & & & & & 4 & 4 & \\
\hline 08 & 4,8 & 220 & 26 & 3,3 & 195 & 15 & 2,2 & 100 & 15 & 8 & & \\
\hline 09 & 5,2 & 230 & 28 & 3,6 & 190 & 21 & 2,5 & 100 & 20 & t & 4 & \\
\hline 10 & 5,6 & 240 & 28 & 3,9 & 190 & 26 & 2,7 & 100 & 21 & & & \\
\hline 11 & 6,0 & 250 & 28 & 4,1 & 190 & 28 & 2,9 & 100 & 25 & 4 & 4 & \\
\hline 12 & 6,0 & 260 & 28 & 4,2 & 185 & 28 & 3,0 & 100 & 26 & 7 & & \\
\hline 13 & 6,0 & 240 & 28 &,+ 1 & 180 & 27 & 3,0 & 100 & 26 & 4 & & \\
\hline 14 & 5,9 & 230 & 28 & 4,0 & 190 & 27 & 2,9 & 100 & 26 & 4 & 4 & \\
\hline 15 & 5,9 & 230 & 28 & 3,8 & 200 & 27 & 2,7 & 100 & 26 & 7 & & \\
\hline 16 & 5,7 & 230 & 28 & 3,6 & 200 & 25 & 2,5 & 100 & 25 & 4 & 4 & \\
\hline 17 & 5,7 & 220 & 28 & 3,3 & 200 & 16 & 2,2 & 100 & 14 & 4 & 4 & \\
\hline 18 & 5,5 & 200 & 28 & & & & & & & 25 & & \\
\hline 19 & 4,8 & 210 & 28 & & & & & & & 14 & & \\
\hline 20 & 3,9 & 220 & 28 & & & & & & & 21 & 7 & \\
\hline 21 & 3,3 & 230 & 28 & & & & & & & 10 & 4 & \\
\hline 22 & 3,3 & 240 & 27 & & & & & & & 22 & & \\
\hline 23 & 3,2 & 250 & 26 & & & & & & & 12 & 4 & \\
\hline
\end{tabular}

APRILE 1953

\begin{tabular}{|c|c|c|c|c|c|c|c|c|c|c|c|c|}
\hline \multirow{2}{*}{ ORA } & \multicolumn{9}{|c|}{ VALORI MEDIANI MENSILI } & \multicolumn{3}{|c|}{ Percentuale Es } \\
\hline & foF2 & $h^{\prime} F 2$ & val & foFl & $h^{\prime} \mathrm{F} 1$ & val & foE & $h^{\prime} E$ & val & tot & $>5$ & $>7$ \\
\hline 00 & 3,3 & 260 & 19 & & & & & & & 16 & 5 & \\
\hline 01 & 3,3 & 275 & 20 & & & & & & & 5 & 5 & \\
\hline 02 & 3,1 & 280 & 22 & & & & & & & & & \\
\hline 03 & 3,3 & 270 & 22 & & & & & & & 13 & 5 & \\
\hline 04 & 3,1 & 260 & 23 & & & & & & & 17 & 4 & 4 \\
\hline 05 & 2,9 & 250 & 25 & & & & & & & 20 & & \\
\hline 06 & 2,8 & 220 & 25 & & & & & & & & & \\
\hline 07 & 4,7 & 230 & 24 & 3.5 & 200 & 7 & 2,2 & 100 & 5 & & & \\
\hline 08 & 5,0 & 270 & 23 & 3,8 & 200 & 22 & 2,7 & 100 & 16 & 13 & 9 & \\
\hline 09 & 5,3 & 270 & 22 & 4,0 & 200 & 24 & 2,8 & 100 & 15 & 13 & 4 & \\
\hline 10 & 5,9 & 260 & 22 & 4,2 & 200 & 23 & 3,0 & 100 & 21 & 27 & 23 & 5 \\
\hline 11 & 6,4 & 280 & 19 & 4,3 & 200 & 17 & 3,2 & 100 & 22 & 22 & 11 & \\
\hline 12 & 6,3 & 270 & 20 & 4,3 & 200 & 19 & 3,3 & 100 & 23 & 20 & 5 & \\
\hline 13 & 6,3 & 280 & 24 & 4,2 & 200 & 24 & 3,2 & 100 & 23 & 12 & 4 & \\
\hline 14 & 6,3 & 260 & 24 & 4,2 & 200 & 24 & 3,1 & 100 & 23 & 8 & 8 & 4 \\
\hline 15 & 6,2 & 270 & 24 & 4,1 & 200 & 23 & 2,9 & 100 & 22 & 8 & 4 & 4 \\
\hline 16 & 6,3 & 250 & 24 & 3,8 & 200 & 24 & 2,8 & 100 & 22 & 17 & & \\
\hline 17 & 6,1 & 240 & 23 & 3,5 & 200 & 18 & 2,5 & 100 & 16 & 9 & 9 & \\
\hline 18 & 6,0 & 220 & 19 & & & & & & & 47 & 5 & \\
\hline 19 & 5,9 & 210 & 17 & & & & & & & 23 & & \\
\hline 20 & 5,7 & 210 & 16 & & & & & & & 31 & 12 & \\
\hline 21 & 5,0 & 215 & 16 & & & & & & & 37 & & \\
\hline 22 &,+ 3 & 220 & 17 & & & & & & & 6 & 6 & \\
\hline 23 & 3,7 & 260 & 17 & & & & & & & 12 & 6 & \\
\hline
\end{tabular}

Osservatorio geofisico di Rocca di Papa dell'I.N.G. - Aprile 1954. 